\title{
Characterization of Uncertainty Contributions in a High-Accuracy PMU Validation System
}

\author{
G. Frigo ${ }^{a, *}$, A. Derviškadića ${ }^{a}$ D. Colangelo ${ }^{b}$, J. P. Braun ${ }^{c}$, M. Paolone ${ }^{a}$ \\ ${ }^{a}$ École Polytechnique Fédérale de Lausanne, 1015 Lausanne, Switzerland \\ ${ }^{b}$ Det Norske Veritas GL, 6812 Arnhem, The Netherlands \\ ${ }^{c}$ Federal Institute of Metrology, 3003 Bern-Wabern, Switzerland
}

\begin{abstract}
The effective deployment of Phasor Measurement Units (PMUs) in Distribution Networks (DNs) requires an enhancement in terms of estimation accuracy beyond the limits of IEEE Std C37.118.1 (IEEE Std), aiming at a Total Vector Error (TVE) in the order of $0.0 \mathrm{x} \%$ in steady-state test conditions. As a consequence, a rigorous metrological characterization of PMU performance requires a validation system whose accuracy is at least one order of magnitude better than the one of the device under test, i.e. it requires a TVE in the order of $0.00 \mathrm{x} \%$ in steadystate test conditions and $0.0 \mathrm{x} \%$ in distorted or dynamic test conditions. In this paper, we consider the hardware and software architecture of a previously published PMU validation system specifically designed for PMUs operating in DNs. In this context, we evaluate the quality of the generated test waveforms, and we carry out a thorough metrological characterization of the uncertainty contributions due to generation, acquisition and synchronization stages. In this sense, the proposed analysis provides a rigorous methodology for the evaluation of such low uncertainty levels, and enables us to identify and discuss the most challenging aspects in the implementation of a high-accuracy PMU validation system.
\end{abstract}

Keywords: Phasor Measurement Unit (PMU), validation, metrological characterization, synchrophasor, IEEE C37.118.1

\section{Introduction}

Electric distribution systems (DNs) are rapidly evolving from passive to active power infrastructures [1]. In order to address this issue, the recent literature has discussed the deployment of Phasor Measurement Units (PMUs) also to DN scenario. Indeed, in the presence of distributed renewable energy

\footnotetext{
*Corresponding author: Guglielmo Frigo

Email address: guglielmo.frigo@epfl.ch (G. Frigo)
} 
sources, PMUs could represent an interesting solution for a wide range of applications, like real-time state estimation, power flow monitoring, fault detection, voltage and frequency control [2].

In general, PMUs are measurement devices capable of providing estimates of voltage and current synchrophasors, frequency and Rate-of-Change-ofFrequency (ROCOF) associated to the power signal fundamental component. These estimates are updated with high reporting rates and synchronized with respect to the Coordinated Universal Time (UTC). PMU technology has been originally conceived for monitoring and protection applications in the highvoltage transmission networks (TNs). Accordingly, the related IEEE Std. C37. $118.1[3]$ and its recent amendment [4], henceforth called IEEE Std, define the requirements in terms of accuracy and latency, with specific reference to TNs.

The uncertainty of PMU estimation is expressed in terms of Total Vector Error (TVE), which accounts for both synchrophasor magnitude and phase, Frequency Error (FE), and ROCOF Error (RFE). In this context, the IEEE Std requires TVE not to exceed 1\%, that corresponds to either a magnitude error of $1 \%$ (with null phase error) or a phase error of $1 \operatorname{mrad}$ (at $50 \mathrm{~Hz}$, with null magnitude error).

In view of PMU applications in DNs, this accuracy level proves to be insufficient and unsuitable: the phase difference between adjacent nodes could be comparable with the IEEE Std uncertainty limit [5], and the observed dynamics could be faster and more complex than the ones provided by the IEEE Std compliance tests [6]. It is thus reasonable to say that a definite enhancement in PMU accuracy is not only recommended, but also required for an effective deployment to DNs. In this regard, we can define a more realistic performance requirement as a TVE in the order of $0 . x \%$ in steady-state conditions [7]. As regards FE, distributed generation might produce transitory frequency oscillations with a period in the order of some seconds [8]. Accordingly, an accuracy level around hundreds $\mu \mathrm{Hz}$ would be recommendable in steady-state conditions. As regards RFE, the recent IEEE Std amendment has significantly relaxed the ROCOF accuracy requirements. Nevertheless, based on current ROCOF-based relays, it is reasonable to require an accuracy level around tens $\mathrm{mHz} / \mathrm{s}$ in steady-state conditions [9].

In the recent years, the literature has proposed many different algorithms for synchrophasor estimation capable of facing that level of accuracy [10]. Nevertheless, in practice, the rigorous metrological characterization of the algorithms' performance still represents an open issue for national metrological institutes, research laboratories and manufacturers.

The compliance certification with respect to the standard requirements has to be performed by comparing the device under test with a reference system whose uncertainty is at least one order of magnitude better. Accordingly, in this case, the PMU validation system must generate test waveforms whose reference synchrophasor, frequency and ROCOF are known with a TVE in the order of $0.0 \mathrm{x} \%$ [11]. It is worth noticing that, since a reference standard for DNs is still missing, we refer to the developed architecture as validation rather than calibration system. In this regard, the International Vocabulary of Metrology 
defines validation as the verification process where the device performance is compared with specified requirements adequate for an intended use [12].

The collaboration between the Distributed Electrical Systems laboratory at École Polytechnique Fédérale de Lausanne (DESL-EPFL) and the Swiss Federal Institute of Metrology (METAS) aims at the development of a high-accuracy PMU validation system capable to deal with this stringent requirement. In [13] we describe the hardware architecture, whereas in [14] we thoroughly characterize the software routine used to define the reference synchrophasors, frequency and ROCOF. In [15] we characterize the uncertainty contribution due to the voltage amplifier stage and evaluate their frequency response over the expected measurement bandwidth. The present paper, instead, presents a thorough characterization of the accuracy limits and the hardware uncertainty contributions inherent in the generation, acquisition and synchronization of the test waveforms. To this end, we compare the reference values provided by the calibrator with ground-truth values characterized by higher and traceable accuracy. In particular, for the purely sinusoidal tests, we compare the system's reference values with the measurements of high-accuracy hardware instrumentation (i.e. multimeter and counter). For the non-sinusoidal tests, in the absence of a reliable groundtruth value, we compare the system's reference values with the parameter values included in the ideal mathematical model.

The paper is organized as follows: in Section 2, we provide a brief overview of the existing calibration systems and derive the motivations of this work; Section 3 describes the hardware and software architecture of the proposed PMU validation system; in Section 4 we evaluate the power quality of the generated test waveforms and we perform the metrological characterization of magnitude, phase and frequency uncertainty; the performance in the IEEE Std tests is thoroughly reported in Section 5 in terms of TVE, FE and RFE; in Section 6 we discuss the capability of reproducing user-defined test conditions, such as voltage unbalanced waveforms and current inrush events; finally, Section 7 provides the closing remarks.

\section{Research Scenario}

In general, the hardware architecture of current PMU calibrators is wellestablished and consists of three main stages: (i) a generation stage that generates the test waveforms and feeds them to the PMUs under test; (ii) an acquisition stage that simultaneously acquires the same test waveforms and processes them in order to define the reference synchrophasor, frequency and ROCOF; (iii) a synchronization stage that triggers both the generation and acquisition stage, and guarantees that the calibrator estimates are aligned to UTC. In this regard, it should be noticed that a poor synchronization of generation and acquisition stages would result in a phase discrepancy between the generated waveforms and the reference values employed for the PMU performance evaluation.

It should be noticed that the inclusion of a simultaneous re-acquisition and processing stage is what distinguish a validation system from a high-accuracy waveform generator. The proposed architecture increases the system complexity, 
but allows for a closed-loop control on the actual parameters of the waveforms supplied to the PMUs under test.

The three stages introduce specific uncertainty contributions in the overall calibration procedure. In this context, the system accuracy depends on the capability of minimizing the uncertainty on synchrophasor magnitude and phase angle introduced by generation and acquisition stages, as well as the timing reference uncertainty inherent in the synchronization stage.

Several versions of PMU calibrators have been recently developed by national metrological institutes $[16,17,15,18]$, research laboratories $[19,20,21,14]$ and manufacturing companies [22, 23]. To the best of authors' knowledge, the reference grade is represented by the calibration systems of the National Institute of Standards and Technology (NIST), Washington, DC and of the Swiss Federal Institute of Metrology (METAS), Bern, CH. In fact, they are capable of performing the IEEE Std compliance verification with a TVE in the order of $0.05 \%$ in static test conditions and the uncertainty on synchrophasor phase angle is limited to $300 \mu \mathrm{rad}$.

The motivation of the present paper, in continuation to the work of NIST and METAS, is the metrological characterization of the most significant uncertainty contributions inherent in modern PMU validation systems, with specific reference to synchrophasor phase angle contributions. In particular, we aim at evaluating the overall uncertainty of the reference values provided by a high-accuracy PMU validation system. This value results from several inter-dependent contributions that are inherent in the measurement process, but can be suitably optimized in order to meet the performance target. With respect to previously published contributions, this paper provides a thorough characterization of a complete validation system in all its main components. The knowledge of the uncertainty limit inherent in each system functionality enables us also to define reasonable performance requirements (e.g. in terms of latency or phase accuracy) for PMUbased applications.

For this analysis, we compare the reference values provided by the validation system with ground-truth values characterized by higher and traceable accuracy. In particular, for the purely sinusoidal tests, we compare the calibrator's reference values with the hardware instrumentation results (i.e. multimeter and counter). For the non-sinusoidal tests, in the absence of a reliable ground-truth value, we compare the calibrator's reference values with the parameter values included in the ideal mathematical model of the reproduced test waveform.

\section{Hardware and Software Architecture}

\subsection{Hardware architecture}

The hardware architecture of the proposed PMU validation system is shown in Fig. 1 and consists of six main elements. From left to right: (i) the GPSreceiver guarantees the alignment with the absolute time reference; (ii) the rubidium atomic clock enhances the short-term variability of the internal timebase, as shown in Section 4.6; (iii) the time-synchronization board receives from 


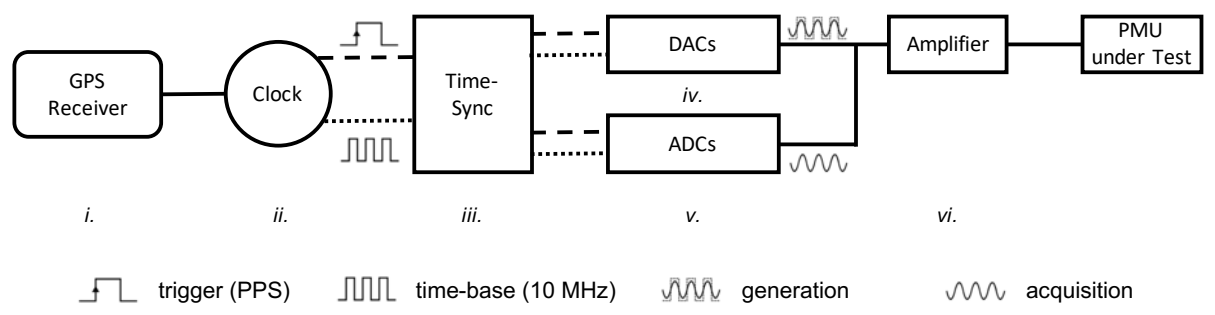

Figure 1: Hardware architecture of the proposed validation system. Generation (DACs) and acquisition (ADCs) boards are aligned to UTC through the same synchronization board, that is disciplined by a GPS-receiver and a Rubidium atomic clock in order to enhance the stability of the internal time-base.

the atomic clock a pulse-per-second (PPS) and a $10 \mathrm{MHz}$ trigger signal and employs them to align the different hardware modules; (iv) the generation board with three digital-to-analog converters (DACs) capable to reproduce three-phase waveforms compliant with IEEE Std specifications; (v) the acquisition board whose analog-to-digital converters (ADCs) re-acquire the waveforms sent to the PMU under test and enable us to extract the reference values of synchrophasor, frequency and ROCOF; (vi) the voltage and current amplifier that increase the generation output range up to $300 \mathrm{Vrms}$ and $32 \mathrm{~A}$, in line with the expected levels in a DN scenario.

The core of the entire validation system is represented by a NI PXI 1042Q (National Instruments, Austin, TX), supplied with three operating modules: the NI PXI-8110 embedded controller, the NI PXI-6682 synchronization board, and the NI PXI-6289 data acquisition board [24]. The internal trigger is provided by the FS725 rubidium oscillator (Stanford Research Systems, Sunnyvale, CA) [25], disciplined by the GPS 169-PCI Radio Clock (Meinberg, Bad Pyrmont) [26]. In this regard, it should be noticed that the rubidium oscillator provides a reduced short-term variability on the $10 \mathrm{MHz}$ time-base signal, whereas the GPS clock guarantees the alignment with respect to UTC and minimizes the long-term variability (see Section 4 for further discussion and experimental validation).

The NI PXI-6289 board enables us to generate and simultaneously re-acquire the three-phase waveforms provided to the PMU under test. In the generation stage, the PMU calibrator employs three 16-bits DACs with an output range of $\pm 10 \mathrm{~V}$ and a sampling frequency of $100 \mathrm{kHz}$. The test waveforms are defined according to the IEEE Std specifications, but can also reproduce additional operating conditions, like phase unbalance or in-rush event, typical of ADN scenarios. In the acquisition stage, the three-phase waveforms are digitized by a multiplexed 18-bits $\mathrm{ADC}$ with an input range of $\pm 10 \mathrm{~V}$ and a sampling frequency of $25 \mathrm{kHz}$. In this regard, it is important to observe that the phase displacement introduced by the multiplexed acquisition has been experimentally characterized and suitably compensated. Any other uncertainty contribution due to internal timing-source is further discussed in Sec. 4.6.

According to the IEEE Std specifications, the typical voltage for testing 

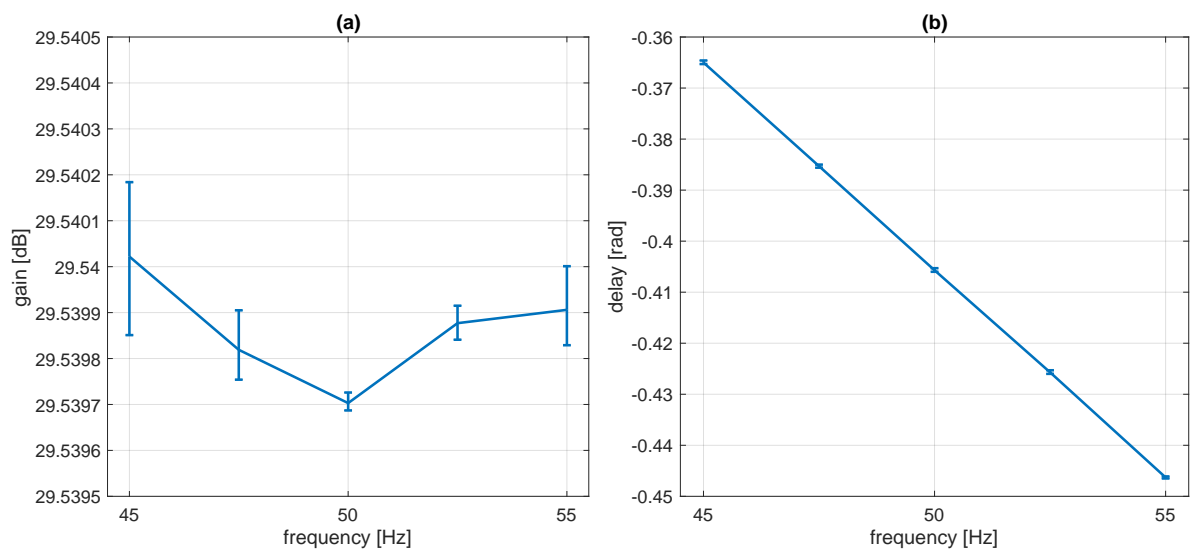

Figure 2: Fequency response of the adopted amplifier in terms of magnitude gain and phase delay in $\mathrm{dB}$ and rad, respectively. The vertical bar represents the measurement variability (ranging from minimum to maximum value) over an observation interval of $2 \mathrm{~s}$.

PMUs is 70 Vrms [27]. In order to meet this requirement, we amplify the DAC output by means of an Omicron CMS 356 amplifier that enables us to provide the PMUs under test with voltage and current waveforms, whose amplitude is limited to $300 \mathrm{Vrms}$ and $32 \mathrm{~A}$, respectively. In this regard, it should be noticed that the accuracy and stability of the amplifier stage still represents an open issue for power system applications and their uncertainty contributions might largely exceed the target performance $[28,29]$. Nevertheless, their frequency response can be carefully characterized and compensated, in order to mitigate the propagation of uncertainty to the final estimation of the reference values [15]. To this end, in Fig. 2, we present the magnitude and phase response and the relative uncertainty variation range $^{1}$ as function of fundamental frequency for the adopted amplifier. Based on the knowledge of this response function, it is possible to suitably define the waveform parameters in order to obtain the desired amplifier output in terms of waveform amplitude and phase. In particular, it is interesting to observe that the magnitude gain is nearly constant with a variation range of $0.0002 \mathrm{~dB}$, whereas the phase response presents a linear trend that can be modeled and compensated as a first-order filter response.

In the proposed architecture, the test waveforms are reproduced by the DACs and simultaneously re-acquired by the ADCs. In this sense, a rigorous characterization of both generation and acquisition stages is necessary. As regards the generation stage, we have experimentally characterized the DAC specifications in an experimental scenario. For this analysis, we employ the DAC to reproduce a sinusoidal waveform in nominal conditions, i.e. with amplitude, frequency and phase equal to $9 \mathrm{~V}, 50 \mathrm{~Hz}$ and $0 \mathrm{rad}$. Coherently with the

\footnotetext{
${ }^{1}$ For this analysis, we quantify the uncertainty as the interval between the minimum and maximum measured value, as shown by the vertical bars in Fig. 2.
} 
aforementioned settings, the sampling rate is set equal to $100 \mathrm{kHz}$. The test waveform is measured by means of high-accuracy hardware instrumentation. On one side, a digital multimeter with a resolution of 8.5 digits determines the waveform amplitude with a measurement uncertainty of $1 \mu \mathrm{V}$. The discrepancy in this case between nominal and measured value is equal to $83 \mu \mathrm{V}$. On the other side, frequency and phase has been measured by means of a time-counter with a resolution of $10 \mathrm{nHz}$, providing a discrepancy of $4.7 \mu \mathrm{Hz}$.

Conversely, the characterization of the ADC stage is carried out in the following Sections. In fact, the non-linear fitting routine employed to infer the reference values can be interpreted also as a way of assessing the metrological characteristics of the acquisition stage. In this regard, it should be noticed that the drift of magnitude and phase accuracy is primarily caused by the ADCs which tend to be sensitive to temperature. In order to circumvent this problem, the systematic errors have to be re-assessed before every set of tests.

\subsection{Software architecture}

According to IEEE Std specifications, the validation system is required to produce test waveforms that are not purely sinusoidal, as they may contain multiple spectral components or time-varying parameters. Along with the test waveforms, the system is required to produce also the corresponding reference values of synchrophasor, frequency, and ROCOF. To this end, it is necessary to develop a measurement procedure capable of extracting this set of parameters in any test condition provided by the IEEE Std.

In the presence of purely sinusoidal waveforms, it would be possible to just characterize the generated test waveforms by means of a multimeter (for the reference amplitude) and a time counter (for the reference phase and frequency). However, in the presence of non-sinusoidal waveforms, the accuracy level of the hardware instrumentation is no more guaranteed, and we have to adopt another identification technique.

To this end, in the proposed architecture, a non-linear least-squares (NLLSQ) fitting algorithm is employed to process the acquired waveform and determines the reference values of synchrophasor, frequency and ROCOF. The choice of this peculiar synchrophasor estimation algorithm is based on its noticeable accuracy, as shown by the performance characterization in [30] $]^{2}$. Table 1 reports the estimation accuracy in terms of TVE in four significant test conditions, like additive Gaussian noise and interference coming from harmonic or interharmonic components (the complete performance evaluation in IEEE Std test conditions is provided in Section V). In this regard, it is worth observing that NL-LSQ satisfies the performance requirement of a TVE in the order of $0.0 \mathrm{x} \%$.

The proposed validation system implements the mathematical model of a generic time-variant noise-less power signal affected by disturbances, defined as

\footnotetext{
${ }^{2}$ The performance discrepancy with the results presented here and in [30], is due to the fact that in [30] the sampling frequency is equal to $100 \mathrm{kHz}$, whereas the present configuration adopts a sampling frequency of $25 \mathrm{kHz}$.
} 
Table 1: NL-LSQ Performance Summary

\begin{tabular}{c|c}
\hline Test & TVE [\%] \\
\hline nominal steady-state & $1.68 \cdot 10^{-4}$ \\
noise (SNR $=80 \mathrm{~dB})$ & $7.80 \cdot 10^{-4}$ \\
harm. dist. (THD $=10 \%)$ & $1.70 \cdot 10^{-4}$ \\
out-of-band (TIHD $=10 \%)$ & $4.02 \cdot 10^{-4}$ \\
\hline
\end{tabular}

follows:

$$
x(t)=A\left(1+\varepsilon_{A}(t)\right) \cdot \cos \left(2 \pi f t+\varphi_{0}+\varepsilon_{\varphi}(t)\right)+\eta(t)
$$

where $A, f$ and $\varphi_{0}$ are the amplitude, frequency and initial phase of the fundamental component, respectively, $\varepsilon_{A}(t)$ and $\varepsilon_{\varphi}(t)$ account for amplitude and phase modulations, and $\eta(t)$ is the contribution of harmonic or inter-harmonic components or transient events not related to the fundamental component.

During the validation process, the user sets the parameters in (1) to reproduce the desired test conditions. Then, the system provides the corresponding waveforms to one or more devices under test and simultaneously re-acquires them. Finally, the NL-LSQ algorithm determines the triplet of reference values:

$$
\mathcal{P}=\left\{\hat{A}, \hat{f}, \hat{\varphi}_{0}\right\}
$$

Based on these results, we can define the synchrophasor associated to the fundamental component as:

$$
X(t)=A_{s} e^{j \Phi_{s}}, \quad A_{s}=\frac{\hat{A}}{\sqrt{2}}, \Phi_{s}=2 \pi \hat{f} t+\hat{\varphi}_{0}
$$

where $A_{s}$ is equal to the root-mean-squared (RMS) magnitude, and $\Phi_{s}$ is the synchrophasor phase angle that accounts for both fundamental frequency and initial phase.

It is worth mentioning that these synchrophasors are specifically time-stamped by the validation system, whilst a phasor data concentrator collects the estimates provided by the PMU(s) under test, and align them based on their timestamp [31]. In this way, it is possible to compare reference and estimated values associated to the same time-stamp and accordingly determine the accuracy of the devices under test.

In the present analysis, we consider an observation interval of $60 \mathrm{~ms}$, as typically adopted by P-class compliant PMUs. In fact, a longer observation interval cannot satisfy the stringent IEEE Std requirements in terms of reporting latency ${ }^{3}$. In the following metrological characterization, the validation system adopts such observation interval, in order to determine its worst-case performance

\footnotetext{
${ }^{3}$ Since synchrophasor estimates are typically referred to the observation interval midpoint, four nominal cycles represent the upper limit to keep latency lower than two times the reporting period (in the present case: $40 \mathrm{~ms}$ ).
} 
and guarantee a fair comparison with the devices under test. Nevertheless, the validation system is not subject to restrictions in terms of measurement latency and a higher level of accuracy can be achieved by enlarging the observation interval length, as shown in [30].

The reporting rate can be adapted to the specific test requirements. Indeed, given the signal model and the parameter estimates, it is possible to retrieve the reference value at any desired time instant. This functionality proves to be particularly useful during the step change tests, where the IEEE Std requires to perform an interleaved analysis of the PMU response in terms of TVE and FE.

\section{Metrological Characterization in Steady-State Conditions}

In this Section, we provide a rigorous metrological characterization of the test waveform accuracy in terms of magnitude, phase and frequency. Once set the generation parameters, we compare the validation system's estimates with the reference values provided by high-accuracy hardware instrumentation. It should be noticed that the present analysis considers only steady-state test signals, in the absence of interfering tones, as the performance of hardware instrumentation is no more guaranteed otherwise [25, 32, 33]. Nevertheless, it is reasonable to say that similar results are obtained in the other test conditions, as the generation procedure is exactly the same and does not depend on the model of the test signal.

In this regard, it should be noticed that the test waveforms are computed in LabVIEW based on (1) and the user-defined parameters. DACs reproduce the resulting sequences of finite-precision numbers ${ }^{4}$, that are successively acquired by ADCs. For this reason, these stages can be said independent from the implemented signal model.

For each parameter configuration, we acquire a time-series with an overall duration of $5 \mathrm{~s}$, and divide it into 248 consecutive observation intervals of $60 \mathrm{~ms}$, partially overlapped by $40 \mathrm{~ms}$ in order to reproduce a reporting rate equal to 50 fps. Assuming a normal distribution (an experimental proof is given by the quantile-quantile plots displayed in Fig. 3), we describe the discrepancy between estimated and reference values in terms of mean and standard deviation. The first one constitutes a systematic contribution and can be compensated in the system's software, whereas the second one is a random contribution and represents the actual uncertainty of the measurement process. In more detail, the validation system collects the PMU estimates and compares them with the corresponding reference values in order to determine magnitude, phase and frequency errors. In this context, given the statistical distribution of the discrepancy between the system reference values and the ground-truth provided by hardware instrumentation,

\footnotetext{
${ }^{4}$ We adopt the Extended Precision data type: the uncertainty contribution is negligible if compared with generation and acquisition stage ones.
} 

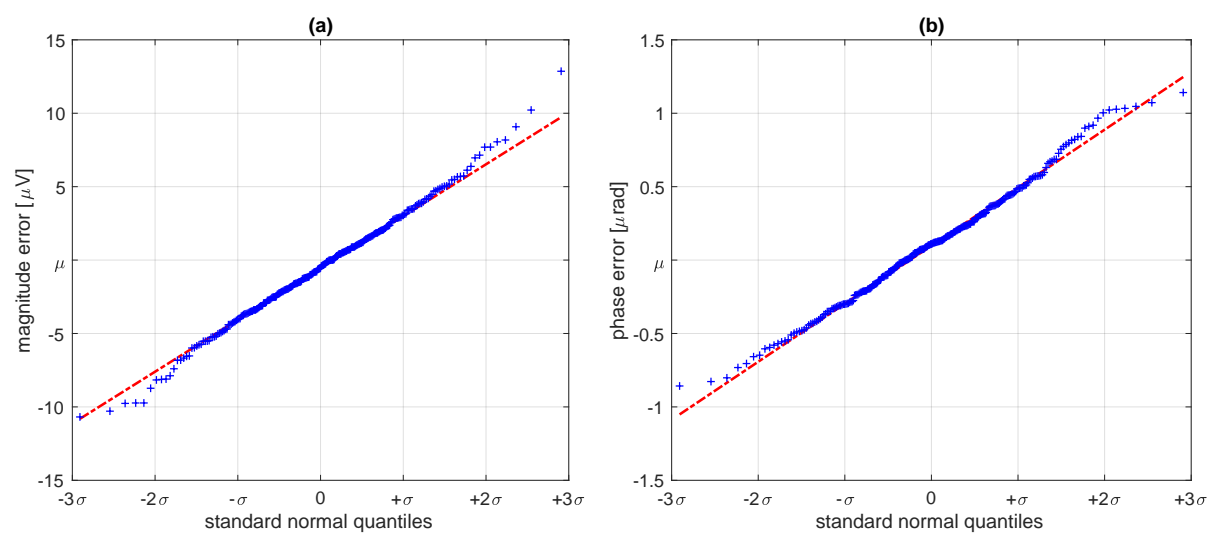

Figure 3: Quantile-quantile plot for magnitude (a) and phase (b) errors in steady-state conditions, with $A=9 \mathrm{~V}, f=50 \mathrm{~Hz}$, and $\varphi_{0}=0 \mathrm{rad}$. Based on the similarity with the ideal trend represented by the red line, we can say that the errors are normally distributed.

it is reasonable to consider the mean discrepancy as an offset due to the combined effect of the system uncertainty sources.

In a calibration scenario, it is reasonable to assume that all the test configurations and waveform parameters are known in advance. It is thus possible to previously characterize the systematic contributions in all the tests (both static and dynamic), and afterwards to carry out the calibration of the PMUs under test. Coherently, the PMU calibration routine can be summarized as follows. First, we characterize the accuracy of the system's reference values (as result of the combined DAC and ADC effects) in terms of systematic (error mean) and random uncertainty (error standard deviation). Then, we connect the validation system to the PMU under test. At the moment of comparing the PMU estimates with the calibrator reference values, the systematic errors are subtracted by the PMU errors as they are fixed offsets. This procedure is replicated for every test, static or dynamic.

Since we consider three-phase waveforms, each parameter configuration provides three slightly different uncertainty values. Unless otherwise indicated, in the following graphs, the bold lines determine the confidence interval between the minimum and maximum standard deviations measured on the three phases, whereas the markers represent the average uncertainty of the system. In this way, it is possible to understand which is the worst-case performance, as required by the IEEE Std, as well as the variation range that can be noticed over the three phases, i.e. a sort of measure of similarity.

\subsection{Power quality of the generated waveforms}

In this subsection, we discuss on the spurious contributions introduced by generation and acquisition stage. First of all, we characterize the uncorrelated noise in terms of signal-to-noise and distortion (SINAD) ratio, and we evaluate the effect of narrow-band components by computing the total harmonic distortion 
(THD) index over the first 50 harmonics. Secondly, we verify that the generated test waveforms are perfectly balanced three-phases signals.

For this analysis, given the signal model in (1), we consider steady-state test conditions (i.e. $\varepsilon_{A}, \varepsilon_{\varphi}, \eta=0$ ). We set the amplitude and initial phase equal to $9 \mathrm{~V}$ and $0 \mathrm{rad}$, respectively, and we vary the fundamental frequency from 45 to $55 \mathrm{~Hz}^{5}$.

In LabVIEW, we compute the SINAD and THD associated to each observation interval. In particular, once detected the spectrum highest peak, an interpolated DFT algorithm estimates the fundamental frequency and RMS amplitude [34]. In this context, SINAD is defined as the ratio between the fundamental energy and the remainder signal energy, expressed in $\mathrm{dB}$, whereas THD is the ratio between the RMS sum of the harmonic tones (up to $50^{\text {th }}$ order) and the RMS amplitude of the fundamental tone. At the same time, we apply the symmetrical components' method to decompose the three-phase signal into its direct, inverse and homopolar sequence. For each $60 \mathrm{~ms}$ observation interval, we compute the energy of inverse and homopolar sequences, and normalize them by the energy of corresponding direct sequence.

In this way, we can infer the distribution of the observed indices in terms of mean value $\mu$ and standard deviation $\sigma$. In Fig. 4 and 5, the markers represent the mean values, whereas the bold lines identify a confidence interval of $\pm 3 \sigma$.

As shown in Fig. 4, in the considered frequency range, SINAD keeps around $93.4 \mathrm{~dB}$, which corresponds to an equivalent number of 15 bits, whereas THD varies between 4 and $6.5 \cdot 10^{-4 \%}$. Both these experimental results confirm that the waveforms are affected by nearly negligible levels of additive noise and distortion. In this regard, it should be noticed that the IEEE Std requires THD and possible distortions coming from interfering components not to overcome $0.02 \%[3]$.

Fig. 5 quantifies the unbalance ratio associated to inverse (blue) and homopolar (red) sequences, as function of the fundamental frequency. Both these contributions exhibit an energy content in the order of some ppm of the direct sequence, whereas the EN Std. 50160 limits the unbalance ratio to $2 \%$.for distribution networks [35]. Accordingly, it is reasonable to say that the proposed system generates test waveforms that adequately approximate a balanced three-phase grid.

We repeat this power quality analysis in all the test conditions provided by the IEEE Std. In a conservative way, we set the waveform parameters according to the worst-case of M-class compliance requirements. For each test, we measure three performance indices: the aforementioned SINAD ratio to evaluate the incidence of noise and distortion, the spurious free dynamic range (SFDR) to determine the ratio between the RMS amplitude of the fundamental and the highest interfering component, and the voltage unbalance factor (VUF) to quantify the entity of the indirect sequence.

\footnotetext{
${ }^{5}$ In this paper, we assume a system nominal frequency of $50 \mathrm{~Hz}$, but equivalent results can be obtained in the $60 \mathrm{~Hz}$-scenario.
} 

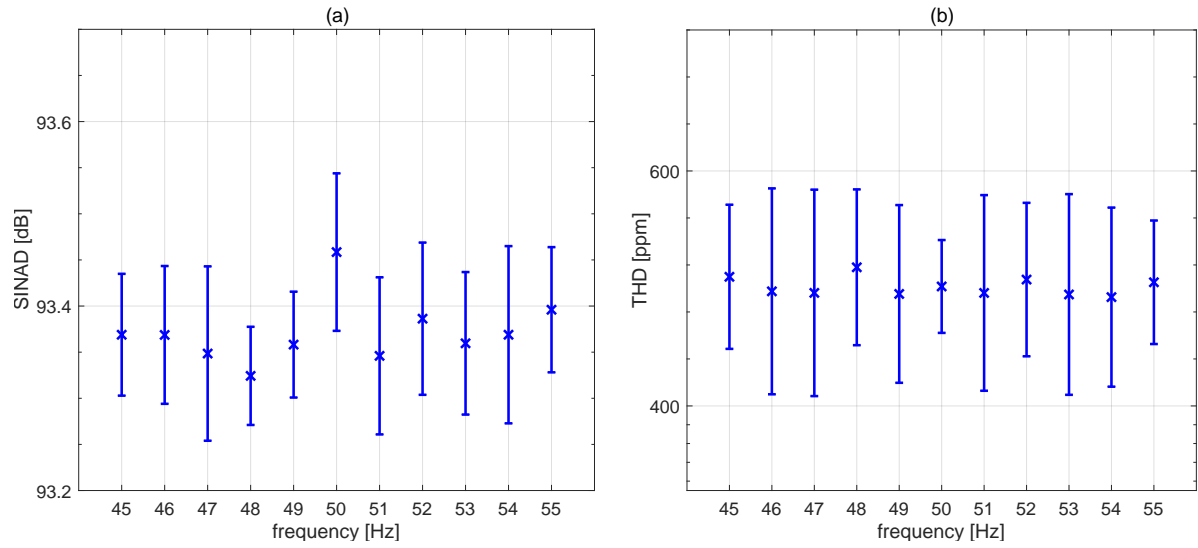

Figure 4: Power quality assessment of the generated test waveform in terms of SINAD (a) and THD (b) in steady-state test conditions, as function of the fundamental frequency. The cross represents the mean value, whereas the bold line determines a confidence interval of three standard deviations.

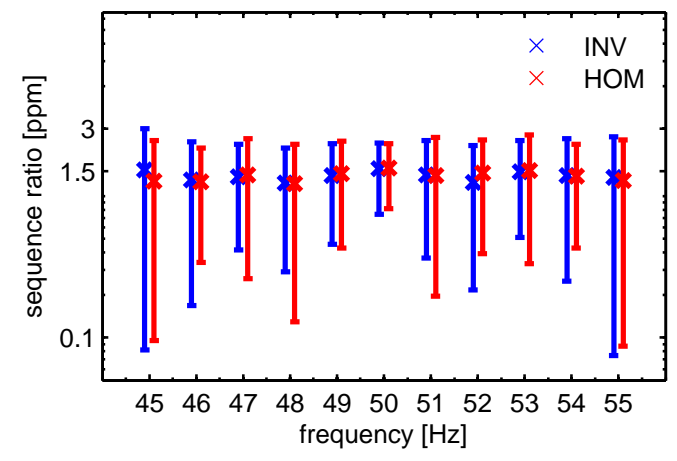

Figure 5: Energy ratio of inverse (blue) and homopolar (red) sequence with respect to direct sequence, in steady-state test conditions, as function of the fundamental frequency. The cross represents the mean value, whereas the bold line determines a confidence interval of three standard deviations. 
Table 2: Power Quality Indices of Generated Test Waveforms

\begin{tabular}{c|c|c|c}
\hline Test & SINAD [dB] & SFDR [dB] & VUF [ppm] \\
\hline steady-state & 93.38 & 110.23 & 1.51 \\
harmonic dist. & 20.04 & 19.96 & 3.11 \\
out-of-band dist. & 20.04 & 19.94 & 2.05 \\
meas. bandwidth & 93.22 & 109.88 & 3.98 \\
frequency ramp & 93.34 & 108.54 & 4.70 \\
step change & 93.33 & 109.36 & 2.54 \\
\hline
\end{tabular}

Table 2 reports the mean value of the considered indices as function of the test condition. In particular, SINAD ratio keeps unaltered around $93 \mathrm{~dB}$, except for harmonic and out-of-band distortion tests, where SINAD is equal to $20 \mathrm{~dB}$. In fact, these two tests require the presence of an interfering component, whose magnitude is equal to $10 \%$ the fundamental magnitude. As regards SFDR, in all the test conditions the index is equal to almost $110 \mathrm{~dB}$, i.e. the noise floor is at least five order of magnitude lower than the fundamental component. Only in the presence of harmonic or out-of-band distortion, SFDR almost reaches 20 dB. Finally, the VUF analysis shows that the mean energy ratio between inverse and direct sequence does not exceed $5 \mathrm{ppm}$. It is thus reasonable to say that the proposed system satisfies the IEEE Std requirements in terms of power quality of the generated waveforms, in both static and dynamic test conditions.

\subsection{Magnitude uncertainty}

We evaluate the uncertainty on the synchrophasor magnitude by comparing the system estimates as result of the NL-LSQ processing on the re-acquired waveforms, with the measurements provided by the 3458A Digital Multimeter (Keysight Technologies, Santa Rosa, CA) with a resolution of 8.5 digits, corresponding to an absolute uncertainty of $\pm 1 \mu \mathrm{V}$. In particular, we adopt the AC Voltage modality with synchronous sub-sampling technique. As shown in Fig. 6, the instrument is triggered by a clock signal derived from the same rubidium atomic clock as the PMU calibrator. We set an input range of $\pm 10 \mathrm{~V}$, an acquisition time window of $1 \mathrm{~s}$, and we compute the average over 5 consecutive readings. In this way, we produce a single measurement for each test but we are capable of reducing the effect of time jitter and measurement noise, and guaranteeing an accuracy of $10 \mathrm{ppm}$ in the frequency range from $1 \mathrm{~Hz}$ to $10 \mathrm{MHz}$ [36, 32]. Based on this ground-truth value, the proposed analysis aims at identifying the 


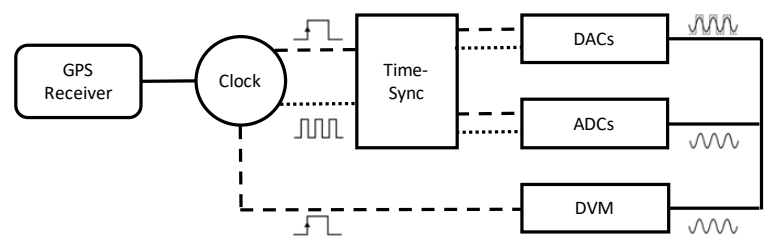

Figure 6: Measurement setup for the assessment of magnitude uncertainty. The generated waveforms are simultaneously sent to the ADC stage and to the digital multimeter (DVM). The synchronization between the two measurement chains is guaranteed by the same trigger provided by the atomic clock.
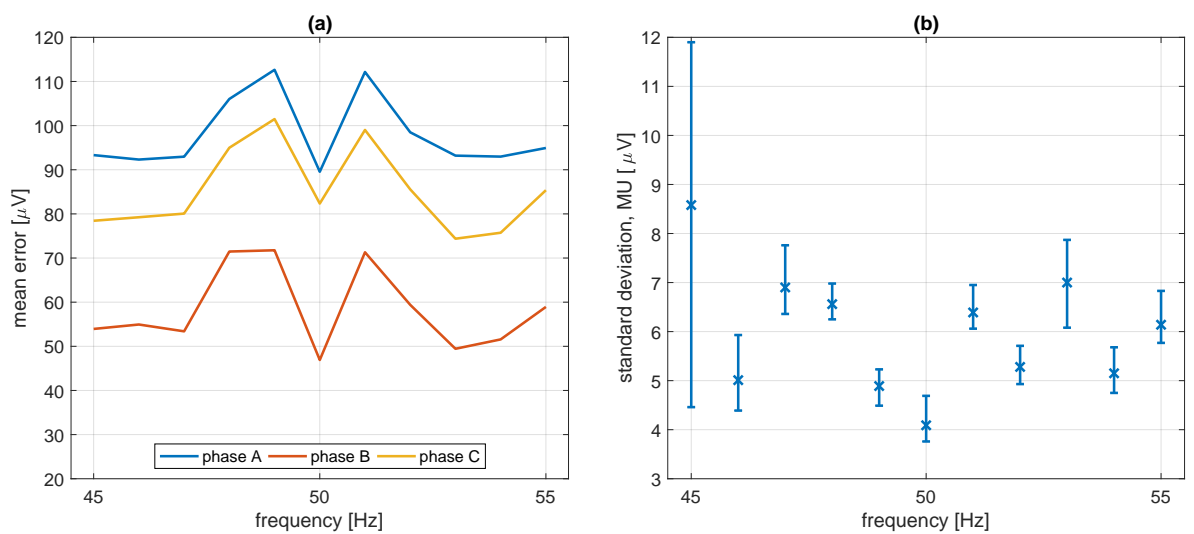

Figure 7: Magnitude estimation error as function of the fundamental frequency, in terms of mean (a) and standard deviation (b). This last increases as the fundamental frequency decreases, with a maximum value of $12 \mu \mathrm{V}$ obtained for $f=45 \mathrm{~Hz}$.

cumulative effect of generation, acquisition and processing stages on the final estimation uncertainty of the synchrophasor magnitude.

For this analysis, we consider a three-phase waveform in steady-state test conditions, and we evaluate the random contribution of synchrophasor magnitude uncertainty, briefly MU, as function of fundamental frequency and magnitude.

In Fig. 7 , we set fundamental amplitude and initial phase equal to their nominal values, i.e. $9 \mathrm{~V}$ and $0 \mathrm{rad}$, whereas we vary fundamental frequency from 45 to $55 \mathrm{~Hz}$ with an incremental step of $1 \mathrm{~Hz}$. In (a) we show the mean error that accounts for the constant uncertainty contribution, whereas in (b) we show the $3 \sigma$ range as representative of the random uncertainty contribution. In the observed frequency range, MU keeps rather stable and does not exceed 12 $\mu \mathrm{V}$. The accuracy performance slightly degrades, as the fundamental frequency decreases, e.g. $45 \mathrm{~Hz}$. As analysed in [30], this is due to the fact that a lower fundamental frequency corresponds to a lower number of periods in the fixed observation interval of $60 \mathrm{~ms}$, resulting in a degradation of the NL-LSQ accuracy.

Fig. 8 shows the normalized MU as function of the fundamental magnitude. 


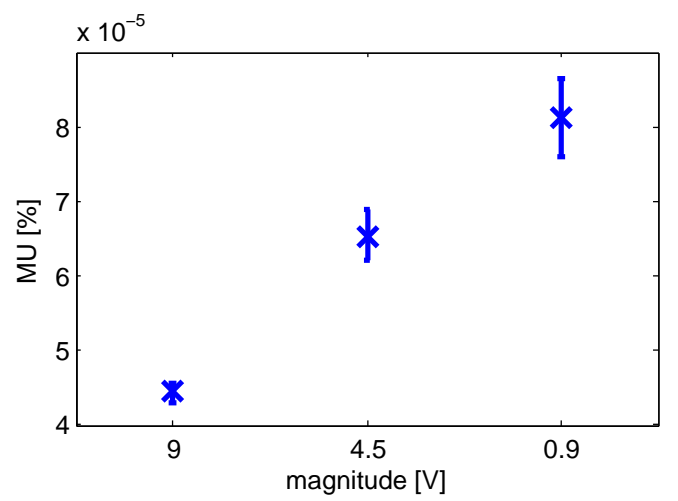

Figure 8: Normalized magnitude uncertainty as function of the fundamental amplitude. It should be noticed that MU keeps below $1 \cdot 10^{-4 \%}$, even when $A$ is decreased up to $10 \%$ of the nominal value.

In the signal model (1), we fix the fundamental frequency and initial phase to $50 \mathrm{~Hz}$ and $0 \mathrm{rad}$, respectively, and we consider three amplitude values, namely $9,4.5$ and $0.9 \mathrm{~V}$, that correspond to 100,50 and $10 \%$ of the nominal value. In the considered range, a slight performance degradation is noticed as the fundamental amplitude decreases. Nevertheless, the percentage error does not exceed $1 \cdot 10^{-4 \%}$.

\subsection{Phase uncertainty}

Given the synchrophasor model in (3), the phase angle $\Phi_{s}$ accounts for both fundamental frequency and initial phase. In this context, a deviation from the nominal system frequency (in our case $50 \mathrm{~Hz}$ ) produces a rotation of the synchrophasor associated to the fundamental component, and can be interpreted as a contribution to the overall phase angle.

As a consequence, the definition of the ground-truth value of the synchrophasor phase angle requires the simultaneous measurement of two quantities, i.e. the fundamental frequency and the initial phase. The adopted measurement testbed is equipped with a time-counter device capable of measuring both frequency and phase, but only one at time. For this reason, in defining the ground-truth $\Phi_{s}$, we need a two-step approach. On one side, we measure the fundamental frequency through the SR620 counter (Stanford Research Systems, Sunnyvale, $\mathrm{CA}$ ), with an acquisition time window of $1 \mathrm{~s}$, and an accuracy in the order of $10 \mathrm{nHz}$ [33]. As in the previous paragraph, the synchronization between the validation system and the analog instrument is guaranteed by the trigger signal provided by the atomic clock (see Fig. 9). On the other side, we define the ground-truth value of fundamental initial phase ${ }^{6}$ by processing the same

\footnotetext{
${ }^{6}$ The counter SR620 cannot provide simultaneous estimates of both frequency and initial phase. Therefore, we need a further measurement procedure to define the reference value for
} 


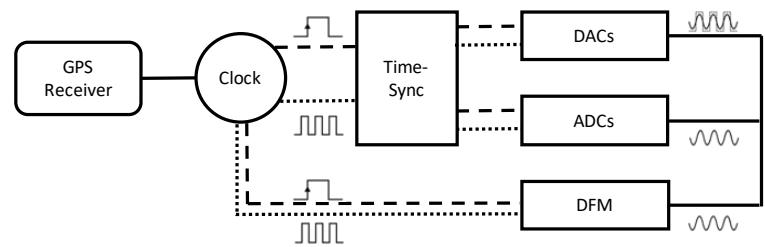

Figure 9: Measurement setup for the assessment of frequency uncertainty. The generated waveforms are simultaneously sent to the ADC stage and to the counter (DFM). The synchronization between the two measurement chains is guaranteed by the same trigger provided by the atomic clock.

acquired waveform with the Enhanced Interpolated DFT (eIpDFT) algorithm. In particular, we consider the entire waveform duration, i.e. $5 \mathrm{~s}$, that enables us to significantly reduce the spectral leakage due to possible harmonic or inter-harmonic components. In addition, the eIpDFT performs a compensation routine that allows for mitigating also the spectral leakage due to the fundamental image at negative frequency. By limiting these uncertainty contribution, the algorithm is expected to provide an accuracy in the order of hundreds nrad given a SINAD around $90 \mathrm{~dB}[34]$. The proposed approach is repeated separately on each phase of the generated three-phase waveform.

Similarly to the analysis of magnitude uncertainty, the metrological characterization considers a three-phase waveform in steady-state test conditions, and evaluates the random contribution of synchrophasor phase uncertainty, briefly PU, as function of fundamental frequency and initial phase.

In the first case, we fix fundamental amplitude and initial phase to $9 \mathrm{~V}$ and $0 \mathrm{rad}$, respectively, and we vary the fundamental frequency from 45 to $55 \mathrm{~Hz}$, with an incremental step of $1 \mathrm{~Hz}$. In this context, Fig. 10 shows the mean and standard deviation of the phase estimation error in (a) and (b), respectively. In particular, PU exhibits a rather symmetrical trend: the uncertainty increases as the fundamental frequency deviates from its nominal value, i.e. $50 \mathrm{~Hz}$. In the considered frequency range, the worst-case performance corresponds to a maximum PU of $0.8 \mu \mathrm{rad}$.

In the second case, instead, we consider the influence of fundamental initial phase in the range $[0, \pi] \mathrm{rad}$, with an incremental step of $\pi / 12 \mathrm{rad}$. As shown in Fig. 11, PU variations are almost negligible and limited to $0.5 \mu \mathrm{rad}$.

\subsection{Frequency and ROCOF}

As further discussed in [30], the proposed validation software assumes the fundamental frequency as a known parameter of the optimization problem solved by the NL-LSQ algorithm. In support of this hypothesis, we assessed the stability of generated waveform frequency over repeated experiments by means of the SR620 counter. Specifically, we have generated single-tone steady state

the fundamental initial phase. 

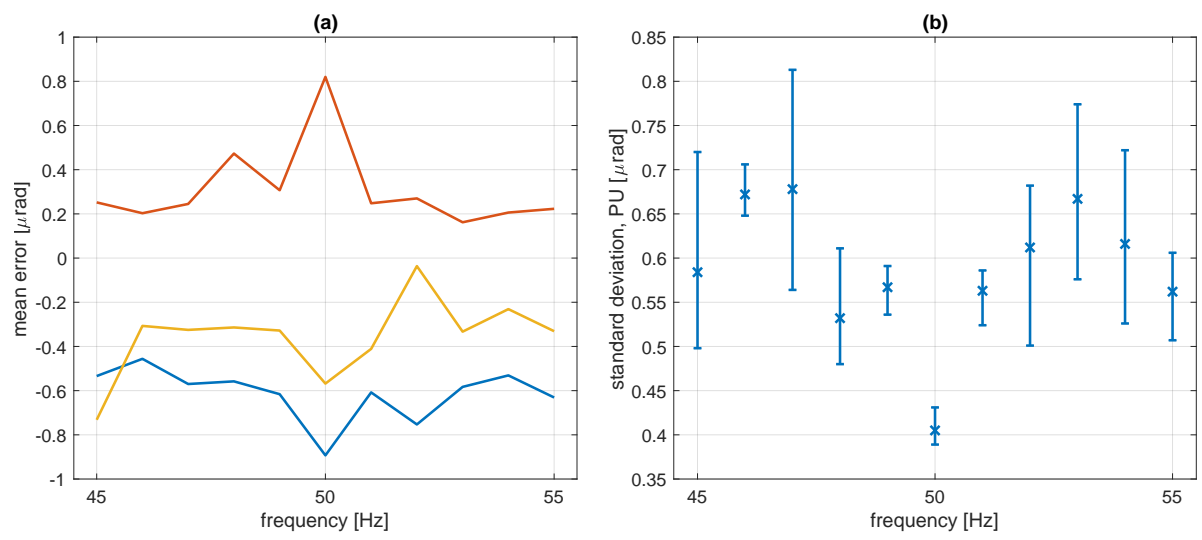

Figure 10: Phase estimation error as function of the fundamental frequency, in terms of mean (a) and standard deviation (b). This last increases as the fundamental frequency deviates from its nominal value, with a worst-case value of $0.85 \mu \mathrm{rad}$.

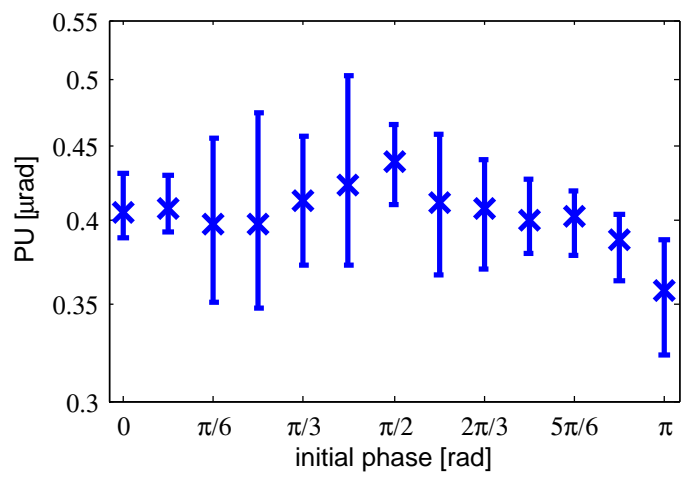

Figure 11: Phase uncertainty as function of the fundamental initial phase. In the entire considered range, PU does not exhibit significant variations, between 0.35 and $0.5 \mu \mathrm{rad}$. 


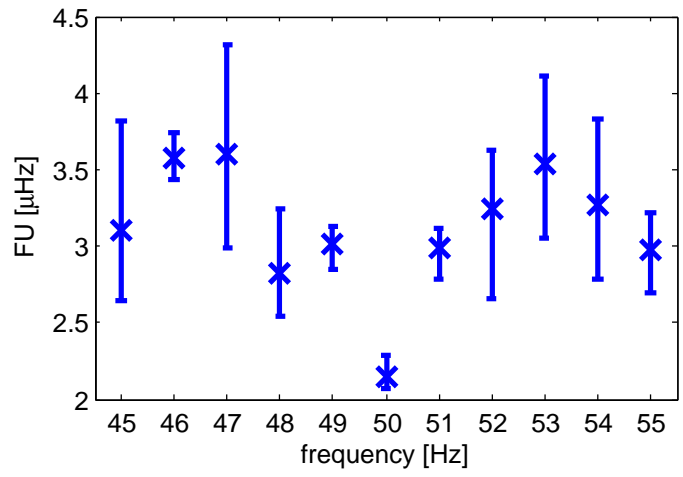

Figure 12: Frequency uncertainty as function of the fundamental frequency. A rather symmetric trend is noticed as the fundamental frequency deviates from its nominal value, $f=50 \mathrm{~Hz}$. In the worst case, FU does not exceed $4.5 \mu \mathrm{Hz}$.

signals by setting frequencies in the range $[45,55] \mathrm{Hz}$. We have noticed that the measured frequency deviates from the value set by the user by some tens of $\mathrm{nHz}$, i.e., comparable with the instrument resolution. Based on the selected signal model and user-defined parameters, the system computes the time-series of the test waveform and sends it to the generation stage, where the DACs reproduce the samples in playback mode. Since the internal time-base stability is guaranteed by the atomic clock, it is reasonable to expect that the sampling process does not introduce significant signal distortions.

The frequency associated to the fundamental component can be defined as the first time-derivative of the synchrophasor phase angle. Therefore, based on PU characterization, we can derive the confidence interval for the estimation of fundamental frequency. The synchrophasor phase angle depends on both fundamental frequency and initial phase. In order to provide a more conservative evaluation of this uncertainty contribution, we assume a zero-valued error on the initial phase, and we interpret the entire PU as only frequency uncertainty (FU).

In Fig. 12, we evaluate FU as function of the fundamental frequency. For this analysis, we set amplitude and initial phase equal to $9 \mathrm{~V}$ and $0 \mathrm{rad}$, respectively, and vary the fundamental frequency from 45 to $55 \mathrm{~Hz}$. At $50 \mathrm{~Hz}, \mathrm{FU}$ is limited to $2.3 \mu \mathrm{Hz}$. In the entire range, we notice a rather symmetric trend: the estimation accuracy degrades as the fundamental frequency deviates from its nominal value. The worst-case performance is obtained at $47 \mathrm{~Hz}$, with FU nearly equal to 4.3 $\mu \mathrm{Hz}$.

The ROCOF uncertainty (RFU) directly descends from FU. Coherently with the IEEE Std formulation, ROCOF is defined as the difference between two consecutive frequency estimates, divided by the reporting time (in our case equal to $20 \mathrm{~ms}$ ). In this context, the worst-case RFU can be computed as:

$$
\mathrm{RFU}=\frac{2 \cdot \mathrm{FU}}{0.02} .
$$


Table 3: Worst-Case Total Vector Errors as function of Characterized System Uncertainties

\begin{tabular}{c|c}
\hline Source & Uncertainty \\
\hline $\begin{array}{c}\text { magnitude } \\
\text { phase angle }\end{array}$ & $\begin{array}{c}12 \mu \mathrm{V} \\
0.8 \mu \mathrm{rad}\end{array}$ \\
\hline \hline Range & TVE [\%] \\
\hline fundamental $[45,55] \mathrm{Hz}$ & $3.56 \cdot 10^{-4}$ \\
disturbance $[5,3000] \mathrm{Hz}$ & $4.02 \cdot 10^{-3}$ \\
\hline
\end{tabular}

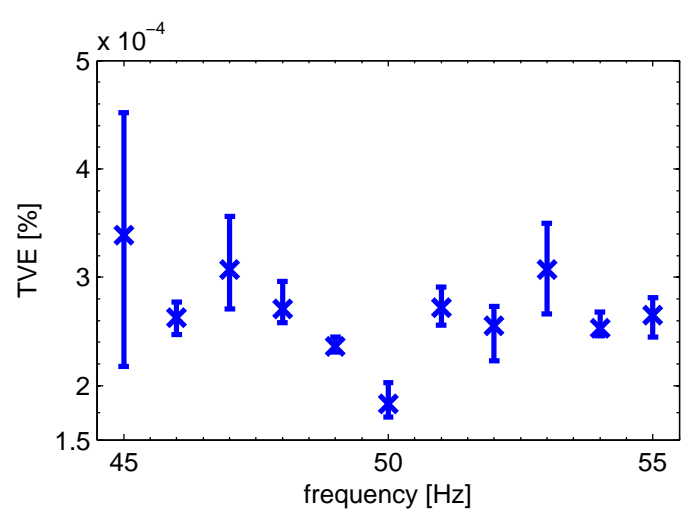

Figure 13: TVE as function of frequency in the range provided for the fundamental component, i.e. from 45 to $55 \mathrm{~Hz}$.

In the considered frequency range, the worst-case RFU is equal to $0.22 \mathrm{mHz} / \mathrm{s}$, whereas, a maximum uncertainty of $0.15 \mathrm{mHz} / \mathrm{s}$ is noticed at $50 \mathrm{~Hz}$.

\subsection{Total Vector Error}

Once characterized the possible sources of uncertainty, we associate a worstcase TVE to the reference values provided by the validation system, by considering a conservative error range of plus/minus three times the standard deviation on both synchrophasor magnitude and phase. In Table 3, we assess the worstcase TVE as function of the fundamental frequency. For this analysis, we consider a three-phase waveform in steady-state test conditions: we set the fundamental amplitude and initial phase equal to their nominal values, $9 \mathrm{~V}$ and $0 \mathrm{rad}$, respectively, while we vary the fundamental frequency from 45 to $55 \mathrm{~Hz}$, with an incremental step of $1 \mathrm{~Hz}$. As shown in Fig. 13, the worst-case TVE does not exceed $5 \times 10^{-4 \%}$.

In the IEEE Std compliance verification, several test conditions require the validation system to generate multi-tone waveforms, i.e. consisting not only of a fundamental component, but also of one or more interfering components. In this sense, it is worth characterizing the system performance in the entire spectral bandwidth that can be interested by harmonic or interharmonic disturbances. 

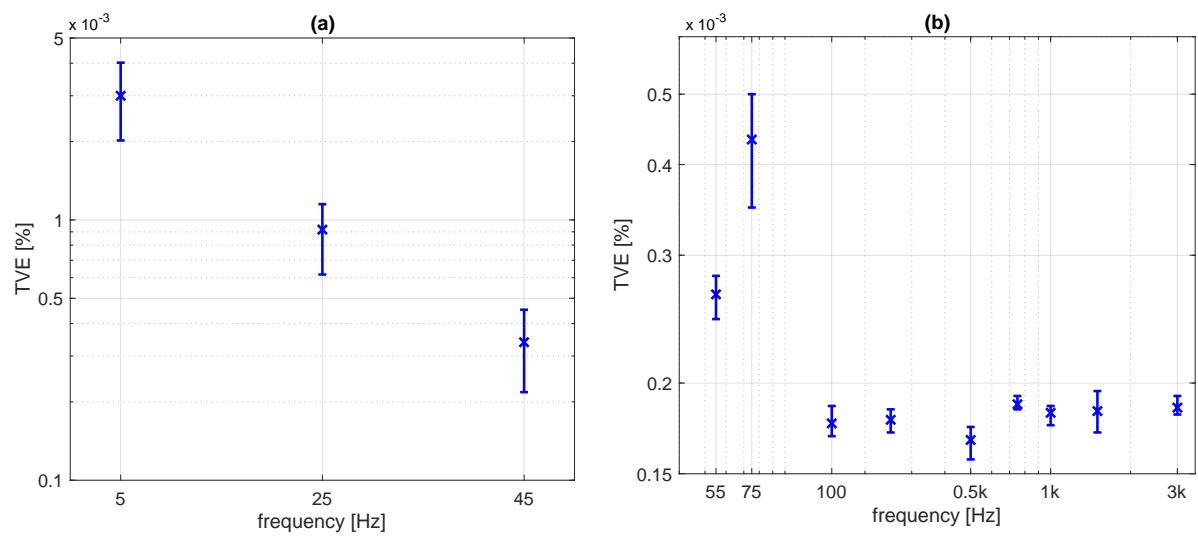

Figure 14: TVE as function of frequency in the range provided for harmonic and interharmonic components, i.e. from 5 to $25 \mathrm{~Hz}$ (a) and from 75 to $3000 \mathrm{~Hz}$ (b).

The upper and lower graphs in Fig. 14 represent the worst-case TVE measured in the range $[5,25]$ and $[75,3000] \mathrm{Hz}$, respectively. In particular, for each test condition we adopt a conservative approach and represent only the highest uncertainty level measured on the three phases, as required by the IEEE Std [3]. In order to provide a term of comparison, in both the graphs we include also the TVE associated to the nearest extreme of the fundamental frequency bandwidth $[45,55] \mathrm{Hz}$. In the upper graph, we observe a degradation of the estimation accuracy due to the fact that lower frequency corresponds to lower number of periods in a given observation interval, and the fitting algorithm accuracy degrades up to a worst-case TVE in the order of $5 \times 10^{-3} \%$. As further discussed in [30], the performance could be easily enhanced by increasing the observation interval length or the sampling frequency, but we prefer to keep the same experimental configuration throughout the entire paper for the sake of congruency. In the lower graph of Fig. 14, the TVE is rather stable around $2 \times 10^{-4 \%}$, i.e. the same accuracy level measured at the nominal frequency of 50 $\mathrm{Hz}$. In the considered range, only at $75 \mathrm{~Hz}$, a reduced performance degradation is noticed, with the worst-case TVE not exceeding $5 \times 10^{-4 \%}$.

This analysis enables us to define a sort of frequency response of the proposed validation system. The experimental results confirm that it guarantees a noticeable level of estimation accuracy not only in the range associated to the fundamental component, but also in the entire spectral bandwidth associated to possible harmonic and interharmonic disturbances.

\subsection{Stability issues}

In the presence of repeated validations, an aspect to be considered is the consistency and steadiness of the estimated reference values. Some uncertainty sources might introduce uncompensated contributions and thus produce erroneous estimates of the reference magnitude and phase. In particular, we focus on two 
synchronization issues: first, the atomic clock jitter increases the phase noise; second, the synchronization delay between generation and acquisition stages results in an imprecise definition of the reference initial phase. In the following paragraphs, we consider each source individually and discuss their effect on the validation results.

Timing uncertainty. The internal timing reference is provided by the rubidium atomic clock, disciplined by the GPS receiver. This hardware configuration enables us to simultaneously reduce both short- and long-term variability. On one side, the GPS receiver provides a PPS signal, characterized by a noticeable long-term stability, and guarantees the alignment with respect to UTC. On the other side, the rubidium atomic clock provides a $10 \mathrm{MHz}$ time-base signal, with reduced short-term variability, that can be used to synchronize all the modules connected to the PXI chassis.

It is worth mentioning that the rubidium atomic clock derives the time-base signal directly from the PPS signal received from the GPS receiver. Thus, if we consider observation interval lengths not lower than $1 \mathrm{~s}$, we can assume that the two signals are perfectly synchronized and locked in phase.

In this context, we provide a quantitative assessment of the performance enhancement guaranteed by the proposed configuration. First, we evaluate the long-term stability of the PPS signal. At METAS laboratories, we compare the PPS signal provided to the PXI with the PPS signal directly derived from UTC-CH. For this analysis, we employ the SR620 counter in time interval modality with an accuracy of 100 ps [33]: the PPS derived form UTC-CH starts the counter, while the PPS routed out by the PXI stops the counter. Fig. 15 presents the time deviation measured over an observation interval of 48 hours. We notice an average deviation in the order of hundreds ns, that represents a systematic contribution due to the length of the antenna cable $(25$ $\mathrm{m}$ ) and can be compensated. Conversely, the standard deviation constitutes the actual contribution to the overall phase uncertainty. Considering a fundamental frequency of $50 \mathrm{~Hz}$, the PPS uncertainty not larger than $12 \mathrm{~ns}$ corresponds to an estimation error on the synchrophasor phase of $4 \mu \mathrm{rad}$.

Then, we assess the short-term stability of the $10 \mathrm{MHz}$ time-base signal. At METAS laboratories, we measure the phase noise as function of the frequency offset with respect to the signal carrier frequency. In Fig. 16 we compare three different configurations: the first one consists of the PXI in free-running mode (blue circles), in the second one the PXI time-base is disciplined by the GPS receiver only (red crosses), the third one considers the proposed hardware configuration, with the rubidium atomic clock, disciplined by the GPS receiver (green squares). It is interesting to observe how the employment of the GPS receiver only produces a reduced enhancement in the long-term stability. Conversely, the rubidium atomic clock guarantees a reduction of the phase noise by almost $30 \mathrm{dBc} / \mathrm{Hz}$ in the entire considered range.

Integrating the phase noise curve between $1 \mathrm{~Hz}$ to $20 \mathrm{MHz}$, we can also calculate the equivalent RMS phase jitter affecting the $10 \mathrm{MHz}$ time-base signal. In the proposed hardware configuration, the employment of the rubidium atomic 


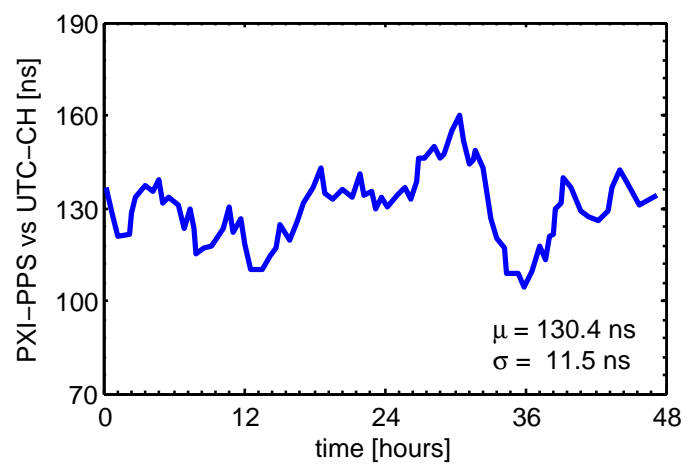

Figure 15: Time deviation between the PPS signal provided to the PXI (PXI-PPS) and the PPS signal directly derived from UTC-CH (UTC-CH). Over an observation interval of two days, the standard deviation is lower than $12 \mathrm{~ns}$, corresponding to a phase uncertainty of 4 $\mu \mathrm{rad}$ at $50 \mathrm{~Hz}$.

clock enables us to limit the jitter in the order of $1 \mathrm{ps}$, that corresponds to a phase uncertainty in the order of hundreds prad, thus negligible if compared with the PPS contribution.

Synchronization uncertainty. Within the proposed architecture, an imprecise internal synchronization of the hardware modules might be translated into a phase discrepancy between generated and acquired waveforms. Within the PMU validation system, the internal time-base and the trigger signals are propagated in a non-ideal and non-deterministic way, that produces a non-negligible jitter between the PXI boards. In particular, the lack of synchronization between generation and acquisition stages produces an initial phase displacement between the waveform acquired and processed by the PMU under test and the reference values provided by the validation system. We characterize the variability of this phenomenon by means of a two-step processing technique, inspired by a METAS procedure [11].

Fig. 17 provides a graphical representation of the proposed technique. In particular, the block diagram consists of two parallel processing schemes. The upper one is related to the phase uncertainty of the acquisition stage only, whereas the lower one accounts for both generation and acquisition stage. From their subtraction, we estimate the initial phase contribution related to the generation stage only.

In the upper scheme, we derive from the $10 \mathrm{MHz}$ time-base a square logic waveform, i.e. a transistor-transistor logic (TTL) signal with a duty cycle of $50 \%$, with a given fundamental frequency $f$. In the following, this signal is referred to as sub-PPS (sPPS), as it is perfectly locked to the PPS signal provided by the GPS antenna. Then, we apply an analog passive low-pass filter with a cut-off frequency of nearly $725 \mathrm{~Hz}$ (the filter consists of a resistor of $10 \mathrm{k} \Omega$ and a capacitor of $0.022 \mu \mathrm{F}$ ). In this way, even though the filtering 


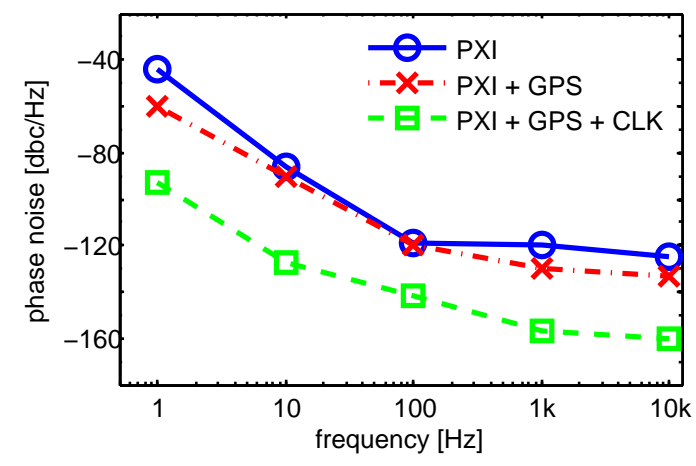

Figure 16: Phase noise obtained with three different configurations: free-running PXI (circles), PXI disciplined by GPS receiver only (crosses), PXI disciplined by atomic clock and GPS receiver (squares). The last one guarantees a significant phase noise reduction over the considered frequency range.

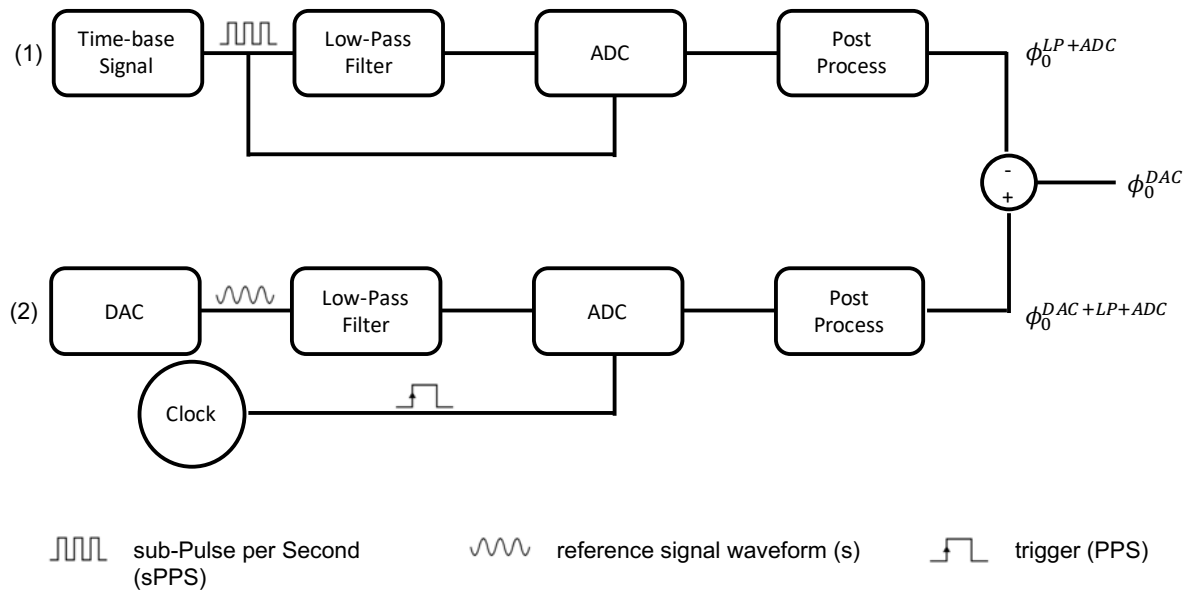

Figure 17: Initial phase displacement due to imprecise synchronization between generation and acquisition stage. In 1, we evaluate the uncertainty due to low-pass filter and acquisition stages. In 2, we determine the uncertainty due also to generation stage. The subtraction provides the single DAC contribution. 
stage introduces a systematic phase contribution, it limits the sPPS spectral bandwidth. Thus, we can acquire the filtered waveform without noticeable aliasing. For this analysis, we adopt a sampling frequency of $25 \mathrm{kHz}$ with an experiment duration of $5 \mathrm{~s}$, we trigger the ADC with the sPPS itself. We divide the acquired time-series into 200 observation intervals of $1 \mathrm{~s}$, partially overlapped in order to reproduce a reporting rate of $50 \mathrm{fps}$. First, we remove the mean value in order to mitigate the spectral contributions caused by DC and low frequency. Then, we estimate the initial phase of the fundamental component by means of the eIpDFT algorithm. By repeating this analysis on each observation interval, we are able to determine the statistical distribution of the sPPS initial phase, in terms of mean and standard deviation, and thus characterize the uncertainty contribution due to low-pass filtering (LP) and acquisition (ADC) stages, $\varphi_{0}^{L P+A D C}$.

In the lower scheme, we employ the validation system to generate a sinusoidal waveform $\mathrm{s}$ in steady-state test conditions, with the same fundamental frequency $f$ as in the upper scheme. For this analysis, amplitude and initial phase are set equal to $9 \mathrm{~V}$ and $0 \mathrm{rad}$, respectively. Then, we apply the same analog lowpass filter, and we trigger the ADC with the PPS signal provided by the GPS antenna. In this sense, the uncertainty on the trigger event is comparable to the one of the upper scheme, as sPPS and PPS are locked by construction. As in the upper scheme, the acquired time-series is divided into 200 observation intervals of $1 \mathrm{~s}$, with a reporting rate of $50 \mathrm{fps}$. We use the eIpDFT algorithm to estimate the initial phase in each observation interval, and thus derive its statistical distribution in terms of mean and standard deviation. In this way, the lower scheme accounts for the uncertainty contribution due to generation (DAC), low-pass filtering (LP), and acquisition (ADC) stages, $\varphi_{0}^{D A C+L P+A D C}$.

Finally, we compute the difference between lower and upper scheme estimates. The resulting distribution accounts only for the generation uncertainty $\varphi_{0}^{D A C}$. Indeed, the contributions due to low-pass filtering and acquisition are perfectly equivalent in upper and lower schemes, and the corresponding synchronization signals (sPPS and PPS) are directly derived by the same timing reference.

Fig. 18 represents the time-domain trend of sPPS and s, in blue and red line, respectively, when the fundamental frequency is set equal to $50 \mathrm{~Hz}$. It is interesting to observe how the LP filtering stage has limited the actual bandwidth of the TTL signal, characterized by smoothed transitions and damped oscillations. On first approximation, the signals seem synchronized as their zerocrossing locations are nearly coincident. Nevertheless, the proposed analysis is intended to determine the non-negligible delay introduced by the internal synchronization between DAC and ADC stages.

In Fig. 19, we present the DFT of the acquired sPPS and s. For the sake of clarity, the observed bandwidth is limited to $300 \mathrm{~Hz}$ and the DFT coefficients have been normalized and expressed in pu. Given a frequency resolution of $1 \mathrm{~Hz}$, it is reasonable to assume that the leakage contributions are almost negligible. Also the odd harmonic terms included in the sPPS spectrum do not introduce significant distortions in the fundamental tone estimation.

We repeat this analysis varying the frequency of sPPS and s waveforms 


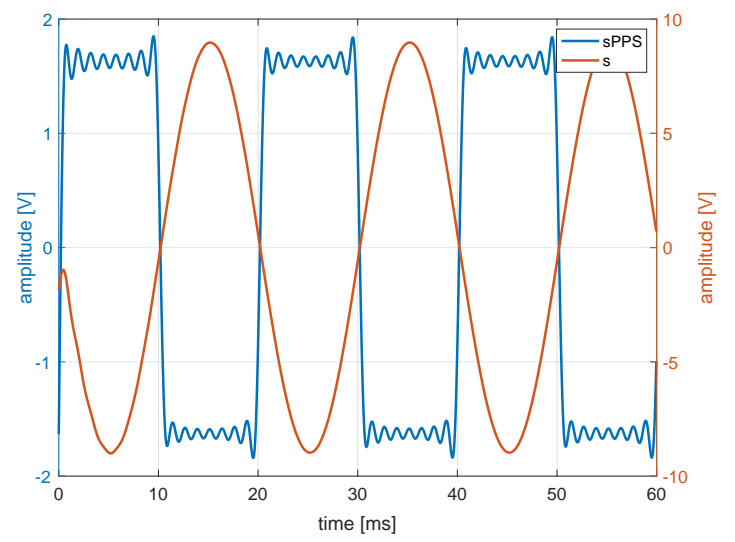

Figure 18: Detail of the signals sPPS (blue) and $\mathrm{s}$ (red), as acquired in the upper and lower scheme of the routine for initial phase characterization. The difference between the corresponding initial phases accounts for the internal synchronization delay between generation and acquisition stages.

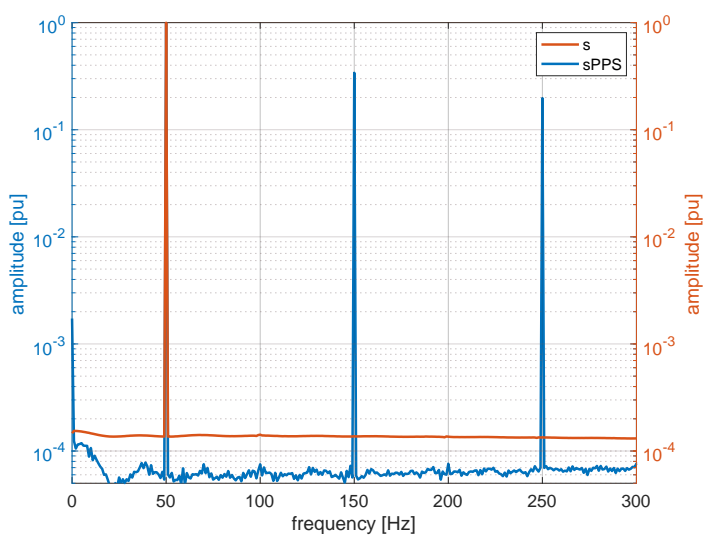

Figure 19: Detail fo the DFT module of the signals sPPS (blue) and s (red), expressed in pu. Nevertheless, the adopted frequency resolution allows for minimizing spectral leakage effects. 


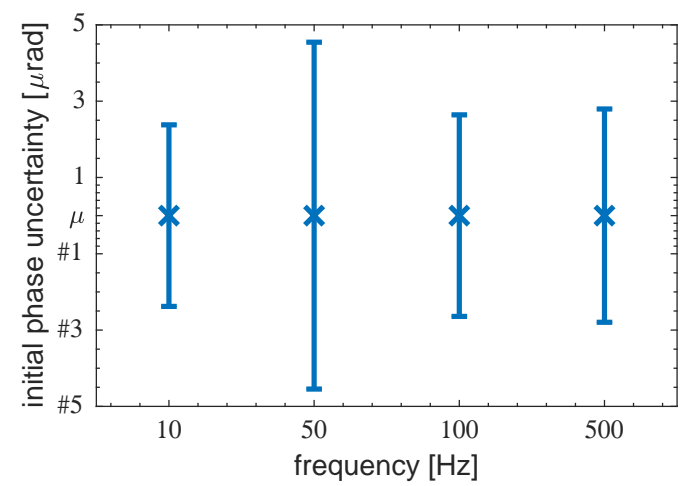

Figure 20: Initial phase uncertainty due to DAC initial phase contribution as function of sPPS and $\mathrm{s}$ frequency.

within the range $[10,500] \mathrm{Hz}$. In particular, we consider frequency values that are integer dividers of $10 \mathrm{MHz}$. In fact, the peculiar configuration of the PXI internal clock requires this constraint to be satisfied, in order to guarantee sufficient stability of the sPPS waveform period. In Fig. 20, we represent the standard deviation of $\varphi_{0}^{D A C}$ as function of sPPS frequency. It is interesting to observe how the synchronization uncertainty ranges from 1.1 to $4.6 \mu \mathrm{rad}$, i.e. it is comparable with the timing uncertainty.

\section{Validation Accuracy in IEEE Std Tests}

In the previous Section, the metrological characterization has involved only steady-state test conditions, in order to guarantee a fair comparison with the estimates provided by the high-accuracy hardware instrumentation. Nevertheless, it is reasonable to assume that the validation system provides similar results in any other test conditions, since the generation, acquisition and fitting procedure keeps unaltered.

In this Section, we characterize the system performance in all the test conditions provided by the IEEE Std. Since the reference values for synchrophasor magnitude, phase, frequency and ROCOF cannot be measured through multimeter or counter (in the dynamic tests), they are set equal to the parameter values set by the user in the generation stage.

Following the same procedure previously described in Section 4, we define the parameters of signal model (1) in order to reproduce the desired test condition, we acquire a time-series of $5 \mathrm{~s}$, and divide it into 248 consecutive observation intervals of $60 \mathrm{~ms}$, with a reporting rate of $50 \mathrm{fps}$. Generation and acquisition stage adopt a sampling frequency of 100 and $25 \mathrm{kHz}$, respectively. Then, we evaluate the synchrophasor magnitude and phase uncertainty, and compute the corresponding worst-case TVE, FE and RFE, as reported in Table 4 as function of the test condition. 
We divide the table into three parts, corresponding to three different classes of test conditions. The upper part accounts for nominal steady-state conditions, with fundamental amplitude, frequency and initial phase set to $9 \mathrm{~V}, 50 \mathrm{~Hz}$ and $0 \mathrm{rad}$, respectively. In this case, TVE is in the order of $2 \cdot 10^{-4 \%}$ and FE does not exceed $2.3 \mu \mathrm{Hz}$.

In the middle part, we consider the M-class requirements for the steady-state compliance verification. First, we evaluate the calibration accuracy as function of the fundamental tone parameters. For this analysis, we vary the fundamental amplitude from 10 to $120 \%$ of its nominal value, and the fundamental frequency from 45 to $55 \mathrm{~Hz}$. In these tests, TVE and FE are limited to $1.85 \cdot 10^{-3 \%}$ and $20.5 \mu \mathrm{Hz}$, respectively.

Then, we consider the effect of harmonic and inter-harmonic distortion. In particular, we reproduce an additive interfering tone, whose amplitude is equal to $10 \%$ of fundamental amplitude. In these tests, we obtain a maximum TVE of $4.05 \cdot 10^{-4 \%}$ and a maximum $\mathrm{FE}$ of $4.2 \mu \mathrm{Hz}$.

The lower part presents the performance of the proposed system in the Mclass requirements for the dynamic compliance verification. In the measurement bandwidth test, we apply a simultaneous modulation of amplitude (AM) and phase (PM), with a modulation frequency of $5 \mathrm{~Hz}$ and a modulation depth of $0.9 \mathrm{pu}$ and $0.1 \mathrm{rad}$, respectively. For the sake of completeness, we also report the accuracy of AM and PM applied separately. The maximum TVE is around $3.6 \cdot 10^{-2 \%}$, and the maximum $\mathrm{FE}$ does not exceed $1.35 \mathrm{mHz}$.

For the frequency ramp test, we reproduce a test waveform of $10 \mathrm{~s}$, and vary the fundamental frequency between 45 to $55 \mathrm{~Hz}$ with a linear rate of $1 \mathrm{~Hz} / \mathrm{s}$. In this test, the worst-case performance corresponds to a maximum TVE of $9.2 \cdot 10^{-2} \%$ and a maximum $\mathrm{FE}$ of $4.9 \mathrm{mHz}$.

Finally, we evaluate the performance under step changes in fundamental amplitude and phase. In particular, we consider variations of $\pm 0.9 \mathrm{pu}$ and $\pm \pi / 10$, in terms of amplitude and phase, respectively. In this case, we obtain a maximum TVE of $4.24 \cdot 10^{-4 \%}$ and a maximum FE of $7.2 \mu \mathrm{Hz}$.

The step change tests are intended to evaluate the PMU response time and reporting latency. In more detail, the response time is defined as the time interval between the instants when the PMU measurement accuracy exceeds and respects again given limit (TVE $\leq 1 \%, \mathrm{FE} \leq 5 \mathrm{mHz}$ ). The reporting latency, instead, is the maximum time interval between the time stamp of the PMU measurement, and the time when the data becomes available at the PMU output. In this regard, the IEEE Std requires to quantify response time and reporting latency with an accuracy not lower than $2 \mathrm{~ms}$ and $100 \mu \mathrm{s}$, respectively.

As regards the response time, the proposed system can be easily employed to carry out an interleaved analysis, where the waveform parameters keep constant and the only step occurrence time is varied with a resolution given by the adopted sampling rate, i.e. $40 \mu \mathrm{s}$ at $25 \mathrm{kHz}$. As regards the reporting latency, instead, the PDC integrated within the validation system enables us to collect the measurement data packets coming from the PMU under test and align them based on their time-stamp [31]. In order to guarantee the required level of accuracy, as soon as the waveform generation begins, we count the clock ticks 
Table 4: Calibration Performance in IEEE Std. C37.118.1 Tests

\begin{tabular}{c|c|c|c}
\hline Test & TVE [\%] & FE $[\mathbf{H z}]$ & RFE $[\mathbf{H z} / \mathbf{s}]$ \\
\hline nominal & $2.03 \cdot 10^{-4}$ & $2.29 \cdot 10^{-6}$ & $2.29 \cdot 10^{-4}$ \\
\hline signal amplitude & $1.84 \cdot 10^{-3}$ & $2.02 \cdot 10^{-5}$ & $2.02 \cdot 10^{-3}$ \\
signal frequency & $3.56 \cdot 10^{-4}$ & $4.31 \cdot 10^{-6}$ & $4.31 \cdot 10^{-4}$ \\
harmonic dist. & $1.74 \cdot 10^{-4}$ & $4.19 \cdot 10^{-6}$ & $4.19 \cdot 10^{-4}$ \\
out-of-band dist. & $4.02 \cdot 10^{-4}$ & $1.50 \cdot 10^{-6}$ & $1.50 \cdot 10^{-4}$ \\
\hline meas. bandwidth & $3.57 \cdot 10^{-2}$ & $1.34 \cdot 10^{-3}$ & $1.34 \cdot 10^{-1}$ \\
ampl. modulation & $2.53 \cdot 10^{-5}$ & $6.40 \cdot 10^{-2}$ & $6.40 \cdot 10^{-3}$ \\
phase modulation & $2.52 \cdot 10^{-2}$ & $1.34 \cdot 10^{-3}$ & $1.34 \cdot 10^{-1}$ \\
frequency ramp & $9.17 \cdot 10^{-2}$ & $4.86 \cdot 10^{-3}$ & $4.86 \cdot 10^{-1}$ \\
step change & $4.24 \cdot 10^{-4}$ & $7.19 \cdot 10^{-6}$ & $7.19 \cdot 10^{-4}$ \\
\hline
\end{tabular}

elapsed until each packet arrives. In this way, we are able to characterize the reporting latency with the resolution of the internal time-base, i.e. $100 \mathrm{~ns}$.

\section{Off-Standard Test Conditions}

The proposed validation system is specifically designed for the characterization of PMU operating in DNs. To this end, in addition to the canonical tests provided by the IEEE Std, the proposed system is also capable of reproducing user-defined test conditions, that better approximate typical DN operating conditions. In this sense, a single constraint has to be satisfied: the user-defined signal model has to be a special case of (1).

In this Section, we focus on two specific cases, namely a voltage unbalanced three-phase waveform and a load inrush event, which typically can occur in distribution networks characterized by fast dynamics and high volatility of RESs.

Voltage unbalance. The voltage characteristics of European public distribution systems are regulated by EN 50160. In terms of voltage unbalance, EN 50160 considers ratio between the average RMS value of negative and positive phase sequence, computed over an observation interval of $10 \mathrm{~min}$. Under normal operating conditions, during a week, this ratio shall not exceed $2 \%$. Accordingly, we reproduce a three-phase waveform, whose single phases are defined as follows:

$$
\begin{aligned}
& x_{A}(t)=9.18 \cdot \cos (2 \pi 50 t+0) \\
& x_{B}(t)=9.00 \cdot \cos (2 \pi 50 t+2.1287) \\
& x_{C}(t)=8.82 \cdot \cos (2 \pi 50 t-2.0951)
\end{aligned}
$$

in order to reproduce a voltage unbalance of $2 \%$ (see Fig. 21). 


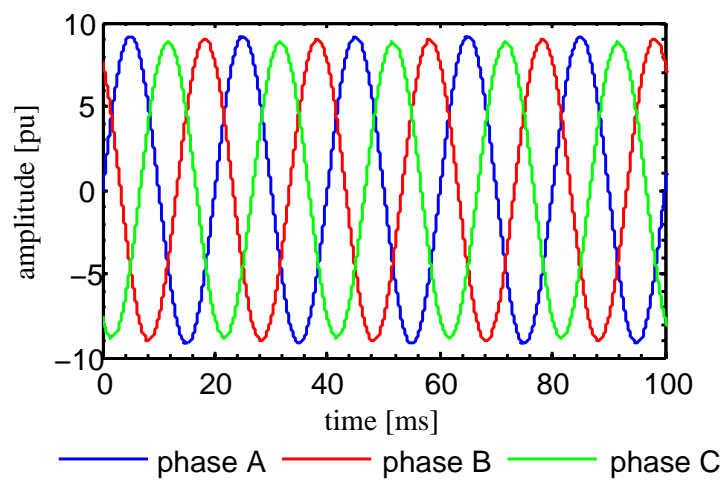

Figure 21: Detail of three-phase unbalanced waveform, compliant with the limits provided by EN 50160. Inverse and homopolar sequence energy correspond to nearly 2 and $0.0003 \%$ of the direct sequence energy, respectively.

Load inrush. As shown in Fig. 22, a load inrush event produces a current waveform with a rapidly increasing amplitude, followed by an exponentiallydecaying trend. The test signal model is defined as follows:

$$
\begin{aligned}
& x(t)=A \cdot \sin \left(2 \pi f t+\varphi_{0}\right)+\eta \\
& \eta(t)=\left(\frac{A_{T}}{1+e^{-k\left(t-T_{T}\right)}}\right) \cdot e^{-\frac{t-T_{T}}{\tau}} \sin (2 \pi f t+\varphi)
\end{aligned}
$$

where the current inrush event is modelled by the additive term $\eta(t)$. The parameters $A_{T}$ and $T_{T}$ determine the transient amplitude and occurrence time, respectively, whereas $\tau$ is the time-constant of the exponential decay. For this analysis, the fundamental amplitude, frequency and initial phase are set equal to 1 p.u., $50 \mathrm{~Hz}$ and $0 \mathrm{rad}$, respectively. The current inrush occurs at $T_{T}=$ $0.495 \mathrm{~s}$ with $\mathrm{k}=10000$, and we set $A_{T}, \tau$ and $\phi$ equal to 6 p.u., 0.087 and 1.53 rad, respectively.

In Table 5, we report the performance in the considered off-standard conditions, in terms of TVE, FE and RFE. In a more conservative approach, for each considered performance index, we report only the maximum value observed on the three phases. In the case of voltage unbalanced waveform, we obtain an accuracy level comparable with the nominal steady-state conditions, with a maximum TVE of $3.70 \cdot 10^{-4} \%$ and a maximum FE of $3.80 \mu \mathrm{Hz}$. On the other side, also in the presence of a severe transient event, like a current inrush, the PMU calibrator is capable of providing accurate estimates, with a maximum TVE in the order of $0.25 \%$ and a maximum FE not exceeding $22 \mu \mathrm{Hz}$.

The proposed system enables us to thoroughly characterize the metrological performance of the PMUs under test. On one side, it performs the entire steadystate and dynamic compliance verification tests, according to the IEEE Std 


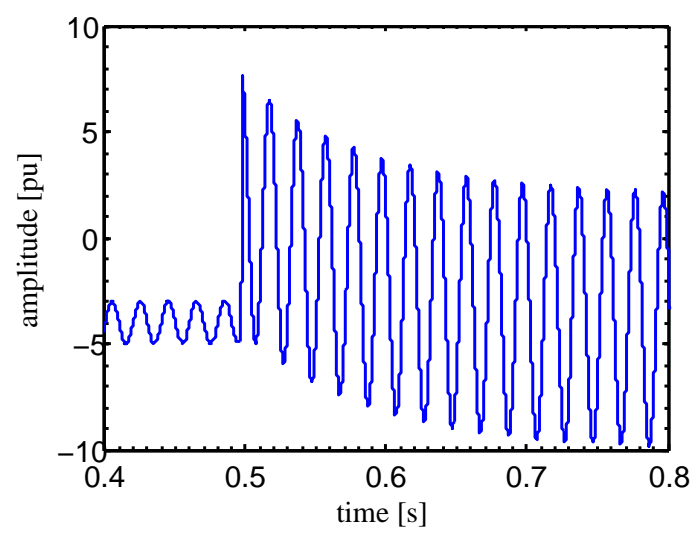

Figure 22: Detail of a current in-rush event, as reproduced by the proposed PMU calibrator.

Table 5: Calibration Performance in Off-Standard Tests

\begin{tabular}{c|c|c|c}
\hline Test & TVE [\%] & FE $[\mathbf{H z}]$ & RFE $[\mathbf{H z} / \mathbf{s}]$ \\
\hline unbalanced & $3.69 \cdot 10^{-4}$ & $3.80 \cdot 10^{-6}$ & $3.80 \cdot 10^{-4}$ \\
inrush & $2.53 \cdot 10^{-1}$ & $2.19 \cdot 10^{-5}$ & $2.19 \cdot 10^{-3}$ \\
\hline
\end{tabular}

requirements. On the other side, the system is also capable of reproducing userdefined test conditions without comparable performance in terms of estimation accuracy. This additional feature could be useful to compare the response of different smart metering devices (such as power meters) to transient events or other off-standard operating conditions.

\section{Conclusion}

This paper presents the metrological characterization of a high-accuracy validation system for PMUs operating in distribution networks. First of all, we provide an overview of the existing PMU calibrators and deduce the motivations of this work: a significant enhancement of the accuracy of the reference values and a rigorous assessment of the uncertainty contributions inherent in modern validation systems.

Then, we described the system hardware and software architecture, and perform a thorough metrological characterization of the test waveform accuracy. First, we evaluate the power quality of the generated test waveforms in terms of SINAD, THD and VUF. Then, we characterize the magnitude, phase and frequency uncertainty by comparing the estimates of the proposed system against the reference values provided by high-accuracy hardware instrumentation. Based on these results, we derive the maximum TVE as function of frequency in the entire spectral bandwidth involved in synchrophasor measurements, i.e. [5, 3000] 
Hz. In nominal conditions, at $50 \mathrm{~Hz}$, TVE is in the order of $2 \cdot 10^{-4 \%}$, whereas the worst performance is obtained at $5 \mathrm{~Hz}$, when TVE does not exceed $5 \cdot 10^{-3} \%$. Finally, we discuss the stability of the estimation accuracy in the presence of repeated experiments. In this context, we evaluate the uncertainty contributions related to the timing reference provided by the atomic clock $(4 \mu \mathrm{rad})$ and the internal synchronization between generation and acquisition stage (4.6 $\mu \mathrm{rad})$.

As the hardware instrumentation accuracy is guaranteed only in stationary non-distorted conditions, the metrological characterization considers only steadystate test waveforms. Accordingly, the performance evaluation in the IEEE Std tests is performed taking as reference the parameter values set by the user in the generation stage. In the steady-state compliance tests, worst-case TVE and $\mathrm{FE}$ are limited to $1.85 \cdot 10^{-3 \%}$ and $20.5 \mu \mathrm{Hz}$, respectively. In the dynamic compliance tests, instead, we obtain a TVE and FE not larger than $9.2 \cdot 10^{-2} \%$ and $4.9 \mathrm{mHz}$, respectively.

The proposed system is also capable of reproducing user-defined test conditions, that better approximate ADNs scenario. In particular, we consider a voltage unbalanced waveform and a load inrush event. This additional feature enables us to compare the response of PMUs or other smart metering device (e.g. power quality meters) to transient events or other off-standard conditions.

Based on the obtained results, it is reasonable to say that the most challenging issue in the implementation of such a high-accuracy validation system is represented by a stable and accurate time-base source and time-dissemination among the several operating blocks. Indeed, the uncertainty due to waveform generation and acquisition can be significantly reduced by means of high-resolution and high-speed DAC and ADC, whilst the contribution of the NL-LSQ processing has been proven to be orders of magnitude lower and thus negligible. On the contrary, the internal synchronization still represents an open issue for a research laboratory that is not supplied with direct link to UTC. In this sense, a rigorous analysis of the phase displacement introduced by incorrect and unstable timebase is necessary in order to mitigate the uncertainty on synchrophasor phase angle, frequency and ROCOF.

\section{Acknowledgment}

The research leading to the results in this paper is part of the European Metrology Research Program (EMRP), which is jointly funded by the EMRP participating countries within EURAMET and the European Union.

\section{References}

\section{References}

[1] M. Kezunovic, J. D. McCalley, T. J. Overbye, Smart grids and beyond: Achieving the full potential of electricity systems, Proceedings of the IEEE 100 (Special Centennial Issue) (2012) 1329-1341. doi:10.1109/JPROC.2012.2187131. 
[2] A. Monti, C. Muscas, F. Ponci, Phasor Measurement Units and Wide Area Monitoring Systems, Academic Press, 2016.

[3] IEEE standard for synchrophasor measurements for power systems, IEEE Std C37.118.1-2011 (Revision of IEEE Std C37.118-2005) (2011) 161doi:10.1109/IEEESTD.2011.6111219.

[4] IEEE standard for synchrophasor measurements for power systems amendment 1: Modification of selected performance requirements, IEEE Std C37.118.1a-2014 (Amendment to IEEE Std C37.118.1-2011) (2014) 125doi:10.1109/IEEESTD.2014.6804630.

[5] C. Muscas, M. Pau, P. A. Pegoraro, S. Sulis, Uncertainty of voltage profile in pmubased distribution system state estimation, IEEE Transactions on Instrumentation and Measurement 65 (5) (2016) 988-998. doi:10.1109/TIM.2015.2494619.

[6] M. Chakir, I. Kamwa, H. L. Huy, Extended c37.118.1 pmu algorithms for joint tracking of fundamental and harmonic phasors in stressed power systems and microgrids, IEEE Transactions on Power Delivery 29 (3) (2014) 1465-1480. doi:10.1109/TPWRD.2014.2318024.

[7] P. Romano, M. Paolone, Dft-based synchrophasor estimation algorithms and their integration in advanced phasor measurement units for the real-time monitoring of active distribution networks, Ph.D. thesis, EPFL, Lausanne (2016).

URL http://desl-pwrs.epfl.ch/cms/site/desl/lang/en/theses

[8] A. Borghetti, C. A. Nucci, M. Paolone, G. Ciappi, A. Solari, Synchronized phasors monitoring during the islanding maneuver of an active distribution network, IEEE Transactions on Smart Grid 2 (1) (2011) 82-91. doi:10.1109/TSG.2010.2094213.

[9] P. Gupta, R. S. Bhatia, D. K. Jain, Active rocof relay for islanding detection, IEEE Transactions on Power Delivery 32 (1) (2017) 420-429. doi:10.1109/TPWRD.2016.2540723.

[10] G. Barchi, D. Macii, D. Petri, Synchrophasor estimators accuracy: A comparative analysis, IEEE Transactions on Instrumentation and Measurement 62 (5) (2013) 963-973. doi:10.1109/TIM.2012.2236776.

[11] J. P. Braun, S. Siegenthaler, The metrological characterization of pmus, in: Workshop on Synchrophasor Estimation Processes for Phasor Measurement Units, EPFL, 2014.

URL http://desl-pwrs.epfl.ch/workshop

[12] International vocabulary of metrology - basic and general concepts and associated terms (vim 3rd edition), Joint Committee for Guides in Metrology (JCGM) (2012).

URL https://www.bipm.org/utils/common/documents/jcgm

[13] D. Colangelo, L. Zanni, M. Pignati, P. Romano, M. Paolone, J. Braun, L. Bernier, Architecture and characterization of a calibrator for pmus operating in power distribution systems, in: 2015 IEEE Eindhoven PowerTech, 2015, pp. 1-6. doi:10.1109/PTC.2015.7232792.

[14] G. Frigo, C. Narduzzi, D. Colangelo, M. Pignati, M. Paolone, Definition and assessment of reference values for pmu calibration in static and transient conditions, in: 2016 IEEE International Workshop on Applied Measurements for Power Systems (AMPS), 2016, pp. 1-6. doi:10.1109/AMPS.2016.7602863.

[15] D. Colangelo, D. Hoogenboom, E. Dierikx, G. Rietveld, G. Frigo, Metrological characterization of a pmu calibrator in the $25 \mathrm{hz}$ to $3 \mathrm{khz}$ range, in: 2017 IEEE Manchester PowerTech, 2017, pp. 1-6. doi:10.1109/PTC.2017.7981109. 
[16] Y. Tang, G. N. Stenbakken, Calibration of phasor measurement unit at nist, in: 2012 Conference on Precision electromagnetic Measurements, 2012, pp. 414-415. doi:10.1109/CPEM.2012.6250979.

[17] J. Braun, C. Mester, Reference grade calibrator for the testing of the dynamic behavior of phasor measurement units, in: 2012 Conference on Precision electromagnetic Measurements, 2012, pp. 410-411. doi:10.1109/CPEM.2012.6250977.

[18] B. Trinchera, D. Serazio, U. Pogliano, Asynchronous phase comparator for characterization of devices and pmu calibrator, in: 2016 Conference on Precision Electromagnetic Measurements (CPEM 2016), 2016, pp. 1-2. doi:10.1109/CPEM.2016.7540459.

[19] R. Garcia-Valle, G. Yang, K. E. Martin, A. H. Nielsen, J. Østergaard, Dtu pmu laboratory development — testing and validation, in: 2010 IEEE PES Innovative Smart Grid Technologies Conference Europe (ISGT Europe), 2010, pp. 16. doi:10.1109/ISGTEUROPE.2010.5638883.

[20] J. Fernandez, The virginia tech calibration system, Master's thesis, Virginia Polytechnic Institute and State University (2011).

URL https://vtechworks.lib.vt.edu/handle/10919/32669

[21] C. Qian, M. Kezunovic, Synchrophasor reference algorithm for pmu calibration system, in: 2016 IEEE/PES Transmission and Distribution Conference and Exposition (T D), 2016, pp. 1-5. doi:10.1109/TDC.2016.7520024.

[22] 6135a/pmucal phasor measurement unit calibration system (2013).

URL http://us.flukecal.com/products

/electrical-calibration/electrical-calibrators/

[23] Q. Guo, F. Chen, R. Piacentini, Virtual-instrumentation-based pmu calibrator for ieee c37.118.1-2011 compliance testing, in: CIGRE Grid of the Future Symposium, 2014. URL http://cigre.wpengine.com/gotf-2014-papers/

[24] 8-slot, universal ac pxi chassis (2014).

URL http://www.ni.com/it-it/support/model.pxi-1042.html

[25] Rubidium frequency standard - fs725, ver 1.3 (2015).

URL www.thinksrs.com/products/FS725.htm

[26] Gps clock for computers (pci/pci-x bus) - gps169pci (2011).

URL www.meinbergglobal.com/english/archive/gps169pci.htm

[27] Ieee guide for synchronization, calibration, testing, and installation of phasor measurement units (pmus) for power system protection and control, IEEE PC37.242/D12, December 2012 (2013) 1-124.

[28] G. Crotti, D. Gallo, D. Giordano, C. Landi, M. Luiso, Industrial comparator for smart grid sensor calibration, IEEE Sensors Journal 17 (23) (2017) 7784-7793. doi:10.1109/JSEN.2017.2724299.

[29] E. Mohns, A. Mortara, H. Cayci, E. Houtzager, S. Fricke, M. Agustoni, B. Ayhan, Calibration of commercial test sets for non-conventional instrument transformers, in: 2017 IEEE International Workshop on Applied Measurements for Power Systems (AMPS), 2017, pp. 1-6. doi:10.1109/AMPS.2017.8078324.

[30] G. Frigo, D. Colangelo, A. Derviškadić, M. Pignati, C. Narduzzi, M. Paolone, Definition of accurate reference synchrophasors for static and dynamic characterization of pmus, IEEE Transactions on Instrumentation and Measurement 66 (9) (2017) 2233-2246. doi:10.1109/TIM.2017.2698709. 
[31] A. Derviškadić, P. Romano, M. Pignati, M. Paolone, Architecture and experimental validation of a low-latency phasor data concentrator, IEEE Transactions on Smart Grid 9 (4) (2018) 2885-2893. doi:10.1109/TSG.2016.2622725.

[32] 3458a multimeter (2014).

URL literature.cdn.keysight.com/litweb/pdf/03458-90014.pdf

[33] Universal time interval counter - sr620 (2006).

URL www.thinksrs.com/products/SR620.htm

[34] P. Romano, M. Paolone, Enhanced interpolated-dft for synchrophasor estimation in fpgas: Theory, implementation, and validation of a pmu prototype, IEEE Transactions on Instrumentation and Measurement 63 (12) (2014) 2824-2836. doi:10.1109/TIM.2014.2321463.

[35] CEI, EN 50160 voltage characteristics in public distribution systems, EN 50160:2010/A1:2015 (2015) 1-14.

[36] R. Lapuh, B. Voljčc, M. Lindič, Evaluation of agilent 3458a time jitter performance, IEEE Transactions on Instrumentation and Measurement 64 (6) (2015) 1331-1335. doi:10.1109/TIM.2015.2408793. 